\title{
Symplectic leaves in real Banach Lie-Poisson spaces
}

\author{
Daniel Beltiţă $\breve{1}^{1}$ and Tudor S. Ratiu ${ }^{2}$
}

\begin{abstract}
We present several large classes of real Banach Lie-Poisson spaces whose characteristic distributions are integrable, the integral manifolds being symplectic leaves just as in finite dimensions. We also investigate when these leaves are embedded submanifolds or when they have Kähler structures. Our results apply to the real Banach Lie-Poisson spaces provided by the self-adjoint parts of preduals of arbitrary $W^{*}$-algebras, as well as of certain operator ideals.

Keywords: Banach Lie-Poisson space; symplectic leaf; characteristic distribution; Kähler manifold; operator ideal; operator algebra
\end{abstract}

MSC 2000: Primary 53D17; Secondary 22E65;58B12;46L30;47L20

\section{Introduction}

This paper studies some geometric properties of the recently introduced Banach Lie-Poisson spaces (see [28]). Every Banach Lie-Poisson space is the predual of some Banach Lie algebra. Two classes of Banach Lie-Poisson spaces will be investigated in this work: preduals of $W^{*}$-algebras and preduals of certain operator ideals.

To explain the geometric questions addressed for these two types of Banach Lie-Poisson spaces, recall that every finite dimensional Poisson manifold has a characteristic generalized distribution whose value at any point is the span of all Hamiltonian vector fields evaluated at that point. The characteristic distribution is always integrable and each of its leaves has two key features: it is an initial symplectic submanifold of the Poisson manifold under consideration that is at the same time a Poisson submanifold (see e.g., 35] or 22]).

If the Poisson manifold is a Lie-Poisson space $\mathfrak{g}^{*}$, where $\mathfrak{g}$ is the Lie algebra of some connected finite dimensional Lie group $G$, it turns out that the integral manifolds of the characteristic distribution of $\mathfrak{g}^{*}$ are just the coadjoint orbits of $G$ with the natural $G$-invariant orbit symplectic structures (see e.g., 35. or 22]). If $G$ is compact, then the coadjoint orbits are $G$-homogeneous embedded Kähler submanifolds of $\mathfrak{g}^{*}$ (see e.g. [17]).

The goal of the present paper is to show that similar phenomena occur in infinite dimensions for large classes of Banach Lie-Poisson spaces. The main results are described in Corollaries [2.10] and 4.6 and in Theorem 5.10 In the case of preduals of $W^{*}$-algebras, weakly symplectic structures on integral

\footnotetext{
${ }^{1}$ Institute of Mathematics "Simion Stoilow" of the Romanian Academy, RO-014700 Bucharest, Romania. Daniel.Beltita@imar.ro.

${ }^{2}$ Centre Bernoulli, École Polytechnique Fédérale de Lausanne, CH-1015 Lausanne, Switzerland. Tudor.Ratiu@epfl.ch.
} 
manifolds of the characteristic distribution have been already constructed in 28 under a certain technical condition. We shall prove in Proposition 2.8 that this condition is always satisfied, for self-adjoint elements, hence all the integral manifolds of the corresponding characteristic distribution are symplectic leaves. On the other hand, in the predual $\mathfrak{S}_{1}$ (trace class operators) of $\mathcal{B}(\mathcal{H})$ (bounded operators) for some complex Hilbert space $\mathcal{H}$, the question of which leaves are actually embedded submanifolds of $\mathfrak{S}_{1}$ was answered in [8] and [9]: they are precisely the leaves containing finite-rank operators. It is noteworthy that a similar characterization of the unitary orbits that are embedded submanifolds of $\mathcal{B}(\mathcal{H})$ had been previously obtained in [1, cf. Theorem 3.1] below. (See 2] for an extension of that characterization to unitary orbits in arbitrary $C^{*}$-algebras.) We shall prove a similar result in the more general setting of operator ideals (see Theorem 5.10). Moreover, we will show that all these embedded submanifolds are actually weakly Kähler homogeneous spaces, thus recovering what happens in finite dimensions for the coadjoint orbits of the compact group $\mathrm{U}(n)$. This circle of ideas is naturally related to the general question of prequantization of infinite dimensional manifolds carrying a closed two form and the problem of finding Banach Lie groups acting naturally on the relevant associated bundles; see 25, 26, 27] for progress in this direction.

\section{Symplectic leaves in preduals of $W^{*}$-algebras}

Throughout the paper, by $C^{*}$-algebra (respectively $W^{*}$-algebra) we actually mean unital $C^{*}$-algebra (respectively unital $W^{*}$-algebra).

Definition 2.1 For every $C^{*}$-algebra $M$, let

$$
\mathcal{P}_{M}:=\left\{p \in M \mid p^{2}=p^{*}=p\right\}
$$

be the set of all orthogonal projections in $M$. We denote by $\mathrm{U}_{M}$ the Banach Lie group of all unitary elements of $M$. Every $u \in \mathrm{U}_{M}$ defines an isometric *-isomorphism

$$
\operatorname{Ad}(u): M \rightarrow M, \quad a \mapsto u a u^{*} .
$$

We also denote by $\mathfrak{u}_{M}$ the Lie algebra of $\mathrm{U}_{M}$, that is,

$$
\mathfrak{u}_{M}:=\left\{a \in M \mid a^{*}=-a\right\} .
$$

Throughout the paper, if $M$ is a $W^{*}$-algebra then $M_{*}$ denotes the predual of $M$ and $M^{*}$ the dual of $M$. An element $\varphi \in M_{*}$ is said to be faithful if $\varphi\left(a^{*} a\right)>0$ whenever $0 \neq a \in M$. This condition is equivalent to the fact that the support of $\varphi$ equals 1 (see Remark 2.5 below).

Recall that a smooth map $f: V \rightarrow W$ between the Banach manifolds $V$ and $W$ is said to be a weak immersion if its tangent map $T_{v} f: T_{v} V \rightarrow T_{f(v)} W$ at any point $v \in V$ is an injective linear bounded map. Note that no assumption about the closedness of the range and its splitting properties are made.

Theorem 2.2 (4]) Let $M$ be a $W^{*}$-algebra and $\varphi \in M_{*}$ faithful. Consider the centralizer of $\varphi$, that is, the sub-W*-algebra

$$
M^{\varphi}=\{a \in M \mid(\forall b \in M) \quad \varphi(a b)=\varphi(b a)\},
$$


and the unitary orbit of $\varphi$,

$$
\mathcal{U}_{\varphi}=\left\{\varphi \circ \operatorname{Ad}(u) \mid u \in \mathrm{U}_{M}\right\} \simeq \mathrm{U}_{M} / \mathrm{U}_{M \varphi},
$$

where $\mathrm{U}_{M^{\varphi}}:=\left\{a \in \mathrm{U}_{M} \mid(\forall b \in M) \quad \varphi(a b)=\varphi(b a)\right\}$ is the unitary group of the centralizer algebra $M^{\varphi}$, that is, the unitary elements of $M^{\varphi}$.

Then the following assertions hold:

(i) $\mathcal{U}_{\varphi} \subseteq M_{*}$.

(ii) The unitary group $\mathrm{U}_{M^{\varphi}}$ of the centralizer algebra $M^{\varphi}$ is a Lie subgroup of $\mathrm{U}_{M}$.

(iii) The unitary orbit $\mathcal{U}_{\varphi}$ has a natural structure of weakly immersed submanifold of $M_{*}$ and $\mathrm{U}_{M}$ acts on it smoothly on the left via $(u, \psi) \in \mathrm{U}_{M} \times \mathcal{U}_{\varphi} \mapsto \psi \circ \operatorname{Ad}\left(u^{-1}\right) \in \mathcal{U}_{\varphi}$.

(iv) The smooth manifold $\mathcal{U}_{\varphi}$ is simply connected.

Proof. (i) This is obvious.

(ii) Note that $\mathrm{U}_{M^{\varphi}}$ is an algebraic subgroup of $\mathrm{U}_{M}$ in the following sense (see Definition 8.9 in [7]):

$$
\mathrm{U}_{M^{\varphi}}=\left\{a \in \mathrm{U}_{M} \mid(\forall p \in \mathcal{P}) \quad p\left(a, a^{-1}\right)=0\right\},
$$

where $\mathcal{P}$ is a set of continuous polynomial functions on $M \times M$. In fact, we may take $\mathcal{P}=\left\{p_{b}\right\}_{b \in M}$, where

$$
p_{b}: M \times M \rightarrow \mathbb{C}, \quad p_{b}(x, y)=\varphi(x b)-\varphi(b x)
$$

whenever $b \in M$; note that the polynomial $p_{b}$ depends only on $x$, but we think of it as a function of $(x, y)$. It is clear that each $p_{b}$ is a continuous linear functional on $M \times M$, and thus a polynomial of degree $\leq 1$.

Now the fact that $\mathrm{U}_{M^{\varphi}}$ is a Lie group with the topology inherited from $\mathrm{U}_{M}$ follows by the main result of [18]; see Theorem 8.12 in [7] for the precise statement in this regard. Furthermore, to prove that $\mathrm{U}_{M^{\varphi}}$ is actually a Lie subgroup of $\mathrm{U}_{M}$, we still have to show that the Lie algebra $\mathfrak{u}_{M^{\varphi}}$ is a split subspace of $\mathfrak{u}_{M}$. The latter fact is a consequence of the fact that, since $\varphi$ is a normal faithful positive form on $M$, there exists a conditional expectation $E$ of $M$ onto $M^{\varphi}$. We recall from Lemma 8.14.6 in 31 that $M^{\varphi}$ equals the fixed-point algebra of the modular group of automorphisms of $M$ associated with $\varphi$. Thus the main theorem of 33 implies that there exists a conditional expectation $E$ from $M$ onto $M^{\varphi}$ satisfying $\varphi \circ E=\varphi$. (Alternatively, the existence of $E$ follows by Remark 2.1 in 3 .)

(iii) The unitary orbit $\mathcal{U}_{\varphi}=\left\{\varphi \circ \operatorname{Ad}(u) \mid u \in \mathrm{U}_{M}\right\}$ through $\varphi \in M_{*}$ is in bijective correspondence with $\mathrm{U}_{M} /\left(\mathrm{U}_{M}\right)_{\varphi}$, where

$$
\left(\mathrm{U}_{M}\right)_{\varphi}:=\left\{u \in \mathrm{U}_{M} \mid \varphi \circ \operatorname{Ad}(u)=\varphi\right\}
$$

is the isotropy subgroup of $\varphi$ under the dual of the action $\operatorname{Ad}$, where $\operatorname{Ad}(u) b:=u b u^{-1}$ for any $b \in M$. It is easily verified that

$$
\left(\mathrm{U}_{M}\right)_{\varphi}=\mathrm{U}_{M \varphi} .
$$

By (ii), $\mathrm{U}_{M^{\varphi}}$ is a Lie subgroup of $\mathrm{U}_{M}$ and thus the set $\mathrm{U}_{M} / \mathrm{U}_{M \varphi}$ has a unique smooth manifold structure making the canonical projection $\mathrm{U}_{M} \rightarrow \mathrm{U}_{M} / \mathrm{U}_{M \varphi}$ a surjective submersion; the underlying 
manifold topology of $\mathrm{U}_{M} / \mathrm{U}_{M \varphi}$ is the quotient topology and $\mathrm{U}_{M}$ acts smoothly on the left on $\mathrm{U}_{M} / \mathrm{U}_{M \varphi}$ by $(u,[v]) \in \mathrm{U}_{M} \times \mathrm{U}_{M} / \mathrm{U}_{M^{\varphi}} \mapsto u \cdot[v]:=[u v]$, where $[v]=v \mathrm{U}_{M^{\varphi}}$ (see Bourbaki 11], Chapter III, §1.6, Proposition 11). Endow the orbit $\mathcal{U}_{\varphi}$ with the manifold structure making this equivariant bijection into a diffeomorphism. It is then easily checked that the inclusion of $\mathcal{U}_{\varphi}$ into $M_{*}$ is a weak immersion.

(iv) See Theorem 2.9 in 4 .

Remark 2.3 (cf. Remark A.2.2 in [20]) There always exist faithful elements in $M_{*}$ provided the predual $M_{*}$ of the $W^{*}$-algebra $M$ is separable.

Remark 2.4 (cf. Proposition 5.1 in [3]) In the setting of Theorem 2.2 assume that $M=\mathcal{B}(\mathcal{H})$ for some complex infinite dimensional Hilbert space $\mathcal{H}$.

Then, for any faithful state $\varphi \in M_{*}$ the orbit $\mathcal{U}_{\varphi}$ is not locally closed in $M_{*}$. Thus, if $M=\mathcal{B}(\mathcal{H})$, the weakly immersed submanifolds occurring in Theorem 2.2 are never embedded submanifolds of $M_{*}$.

Remark 2.5 (cf. Section 5.15 in [32]) Let $M$ be a $W^{*}$-algebra and $0 \leq \varphi \in M_{*}$. Define the support of $\varphi$ by

$$
p:=\mathbf{s}(\varphi):=1-\sup \left\{q \in \mathcal{P}_{M} \mid \varphi(q)=0\right\} \in \mathcal{P}_{M}
$$

The support of $\varphi$ has the following properties:

(i) $(\forall x \in M) \quad \varphi(x)=\varphi(x p)=\varphi(p x)=\varphi(p x p)$.

(ii) If $0 \leq x \in M$ and $\varphi(x)=0$ then $p x p=0$. In particular, $\left.\varphi\right|_{p M p} \in(p M p)_{*}$ is faithful on the $W^{*}$-algebra $p M p$.

For later reference we also note that we have

$$
\left(\forall u \in \mathrm{U}_{M}\right) \quad \mathbf{s}\left(\operatorname{Ad}(u)^{*} \varphi\right)=u^{-1} \mathbf{s}(\varphi) u
$$

since for each $q \in \mathcal{P}_{M}$ the condition $\varphi\left(u q u^{-1}\right)=0$ is equivalent to $u q u^{-1} \leq 1-\mathbf{s}(\varphi)$, hence to $q \leq 1-u^{-1} \mathbf{s}(\varphi) u$.

Remark 2.6 (cf. Section 5.17 in 32 ) Let $M$ be a $W^{*}$-algebra and $\varphi \in M_{*}$ such that $\varphi=\varphi^{*}$, in the sense that $\varphi\left(x^{*}\right)=\overline{\varphi(x)}$ for all $x \in M$. Then there exist $\varphi_{1}, \varphi_{2} \in M_{*}$ uniquely determined by the conditions:

(i) $\varphi=\varphi_{1}-\varphi_{2}$

(ii) $\varphi_{1} \geq 0$ and $\varphi_{2} \geq 0$, and

(iii) $\mathbf{s}\left(\varphi_{1}\right) \mathbf{s}\left(\varphi_{2}\right)=0$.

Lemma 2.7 Let $M$ be a $W^{*}$-algebra, $0 \leq \varphi \in M_{*}, p:=\mathbf{s}(\varphi), \varphi_{p}:=\left.\varphi\right|_{p M p} \in(p M p)_{*}$, and denote, as before,

$$
\mathrm{U}_{M \varphi}=\left\{u \in \mathrm{U}_{M} \mid \operatorname{Ad}(u)^{*} \varphi=\varphi\right\}
$$


Then

$$
\begin{aligned}
\mathrm{U}_{M^{\varphi}} & =\left\{u \in \mathrm{U}_{M} \mid p u=u p, \text { pup } \in \mathrm{U}_{(p M p)^{\varphi_{p}}}\right\} \\
& =\left\{\left(\begin{array}{cc}
u_{1} & 0 \\
0 & u_{2}
\end{array}\right) \in \mathrm{U}_{p} \mid u_{1} \in \mathrm{U}_{(p M p)^{\varphi_{p}}}, u_{2} \in \mathrm{U}_{(1-p) M(1-p)}\right\},
\end{aligned}
$$

where

$$
\mathrm{U}_{p}:=\left\{u \in \mathrm{U}_{M} \mid p u=u p\right\}=\left\{\left(\begin{array}{cc}
u_{1} & 0 \\
0 & u_{2}
\end{array}\right) \mid u_{1} \in \mathrm{U}_{p M p}, u_{2} \in \mathrm{U}_{(1-p) M(1-p)}\right\}
$$

and the $2 \times 2$ matrix is written with respect to the orthogonal decomposition $1=p+(1-p)$.

Proof. Since

$$
\left\{u \in \mathrm{U}_{M} \mid p u=u p\right\}=\left\{\left(\begin{array}{cc}
u_{1} & 0 \\
0 & u_{2}
\end{array}\right) \mid u_{1} \in \mathrm{U}_{p M p}, u_{2} \in \mathrm{U}_{(1-p) M(1-p)}\right\}
$$

it follows that $\mathrm{U}_{p}$ is a Lie subgroup of $\mathrm{U}_{M}$. For all $u \in \mathrm{U}_{M \varphi}$ we have $u^{-1} \mathbf{s}(\varphi) u=\mathbf{s}(\varphi)$ by formula (2.1) in Remark 2.5 Thus, since $p=\mathbf{s}(\varphi)$, we get

$$
\mathrm{U}_{M^{\varphi}} \subseteq \mathrm{U}_{p} .
$$

We now come back to the proof of the desired conclusion. For any $u \in \mathrm{U}_{M}$ we have

$$
(\forall x \in M) \quad \varphi\left(u x u^{-1}\right)=\varphi(x) \Longleftrightarrow(\forall x \in M) \quad \varphi\left(p u x u^{-1} p\right)=\varphi(p x p)
$$

by Remark 2.5(i). Hence for $u \in \mathrm{U}_{p}$ (that is, $u p=p u$ ) we have

$$
(\forall x \in M) \quad \varphi\left(u x u^{-1}\right)=\varphi(x) \Longleftrightarrow(\forall x \in M) \quad \varphi\left((p u p)(p x p)\left(p u^{-1} p\right)\right)=\varphi(p x p) .
$$

Next note that, since $u p=p u$, it follows that $p u^{-1} p$ is just the inverse of $u_{1}:=p u p \in \mathrm{U}_{p M p}$. Thus the above equivalence shows that, for $u=\left(\begin{array}{cc}u_{1} & 0 \\ 0 & u_{2}\end{array}\right) \in \mathrm{U}_{p}$ as above, we have

$$
u \in \mathrm{U}_{M^{\varphi}} \Longleftrightarrow u_{1} \in \mathrm{U}_{(p M p)^{\varphi_{p}}},
$$

and the desired conclusion is proved.

Proposition 2.8 Let $M$ be a $W^{*}$-algebra, $\varphi \in M_{*}$ such that $\varphi=\varphi^{*}$, and

$$
\mathrm{U}_{M^{\varphi}}=\left\{u \in \mathrm{U}_{M} \mid \operatorname{Ad}(u)^{*} \varphi=\varphi\right\} .
$$

Then $\mathrm{U}_{M \varphi}$ is a Lie subgroup of $\mathrm{U}_{M}$.

Proof. Let $\varphi=\varphi_{1}-\varphi_{2}$ as in Remark[2.6] and denote $p_{1}=\mathbf{s}\left(\varphi_{1}\right), p_{2}=\mathbf{s}\left(\varphi_{2}\right)$, so that $p_{1} p_{2}=p_{2} p_{1}=0$. We will prove that

$$
\mathrm{U}_{M^{\varphi}}=\mathrm{U}_{M^{\varphi}} \cap \mathrm{U}_{M^{\varphi_{2}}} .
$$


The inclusion $\supseteq$ is obvious. Now let $u \in \mathrm{U}_{M^{\varphi}}$. Then

$$
\varphi=\operatorname{Ad}(u)^{*} \varphi=\operatorname{Ad}(u)^{*} \varphi_{1}-\operatorname{Ad}(u)^{*} \varphi_{2} .
$$

Moreover, it is clear that $\operatorname{Ad}(u)^{*} \varphi_{j} \geq 0$ and $\mathbf{s}\left(\operatorname{Ad}(u)^{*} \varphi_{j}\right)=u^{-1} p_{j} u$ (by (2.1) in $\operatorname{Remark}$ 2.5) for $j=1,2$, hence $\mathbf{s}\left(\operatorname{Ad}(u)^{*} \varphi_{1}\right) \mathbf{s}\left(\operatorname{Ad}(u)^{*} \varphi_{2}\right)=0$. It then follows from the uniqueness assertion in Remark 2.6 that $\operatorname{Ad}(u)^{*} \varphi_{j}=\varphi_{j}$ for $j=1,2$, hence $u \in \mathrm{U}_{M^{\varphi_{1}}} \cap \mathrm{U}_{M^{\varphi_{2}}}$ as desired.

Next denote $p_{3}=1-p_{1}-p_{2}$, so that $p_{j} \in \mathcal{P}_{M}$ and $p_{i} p_{j}=0$ for $1 \leq i, j \leq 3$, and $p_{1}+p_{2}+p_{3}=1$. According to Lemma 2.7 we have $\mathrm{U}_{M^{\varphi_{p_{j}}}} \subseteq\left\{u \in \mathrm{U}_{M} \mid u p_{j}=p_{j} u\right\}$ for $j=1,2$, hence

$$
\begin{aligned}
\mathrm{U}_{M^{\varphi_{1}}} \cap \mathrm{U}_{M^{\varphi_{2}}} & \subseteq\left\{u \in \mathrm{U}_{M} \mid u p_{j}=p_{j} u \text { for } j=1,2,3\right\} \\
& \simeq\left\{\left(\begin{array}{ccc}
u_{1} & 0 & 0 \\
0 & u_{3} & 0 \\
0 & 0 & u_{2}
\end{array}\right) \mid u_{j} \in \mathrm{U}_{p_{j} M p_{j}} \text { for } j=1,2,3\right\} .
\end{aligned}
$$

Lemma 2.7 actually shows that

$$
\begin{aligned}
\mathrm{U}_{M^{\varphi_{p_{1}}}} & =\left\{u \in \mathrm{U}_{M} \mid p_{1} u=u p_{1}, p_{1} u p_{1} \in \mathrm{U}_{\left(p_{1} M p_{1}\right)^{\varphi_{p_{1}}}}\right\} \\
& \simeq \mathrm{U}_{M^{\varphi_{p_{1}}}} \times \mathrm{U}_{\left(1-p_{1}\right) M\left(1-p_{2}\right)} \\
& =\mathrm{U}_{M^{\varphi_{p_{1}}}} \times \mathrm{U}_{\left(p_{3}+p_{2}\right) M\left(p_{3}+p_{2}\right)}
\end{aligned}
$$

and similarly

$$
\begin{aligned}
\mathrm{U}_{M^{\varphi} p_{2}} & =\left\{u \in \mathrm{U}_{M} \mid p_{2} u=u p_{2}, p_{2} u p_{2} \in \mathrm{U}_{\left(p_{2} M p_{2}\right)^{\varphi_{p_{2}}}}\right\} \\
& \simeq \mathrm{U}_{\left(p_{1}+p_{3}\right) M\left(p_{1}+p_{3}\right)} \times \mathrm{U}_{M^{\varphi_{2}}} .
\end{aligned}
$$

Hence

$$
\begin{aligned}
\mathrm{U}_{M^{\varphi_{1}}} \cap \mathrm{U}_{M^{\varphi_{2}}} & =\left\{u \in \mathrm{U}_{M} \mid p_{j} u=u p_{j}, p_{j} u p_{j} \in \mathrm{U}_{\left(p_{j} M p_{j}\right)^{\varphi_{j}}} \text { for } j=1,2\right\} \\
& \simeq \mathrm{U}_{M^{\varphi_{p_{1}}}} \times \mathrm{U}_{p_{3} M p_{3}} \times \mathrm{U}_{M^{\varphi_{p_{2}}}} \\
& \simeq\left\{\left(\begin{array}{ccc}
u_{1} & 0 & 0 \\
0 & u_{3} & 0 \\
0 & 0 & u_{2}
\end{array}\right) \mid u_{3} \in \mathrm{U}_{p_{3} M p_{2}}, u_{j} \in \mathrm{U}_{M^{\varphi_{j}}} \text { for } j=1,2\right\} .
\end{aligned}
$$

Now $\mathrm{U}_{M^{p_{j}}}$ is a Lie subgroup of $\mathrm{U}_{p_{j} M p_{j}}$ by Theorem 2.2(ii) since $\varphi_{p_{j}}=\left.\varphi\right|_{p_{j} M p_{j}}$ is faithful for $j=1,2$ by Remark 2.5(ii). Hence the above isomorphism shows that $\mathrm{U}_{M^{\varphi_{1}}} \cap \mathrm{U}_{M^{\varphi_{2}}}$ is a Lie subgroup of $\mathrm{U}_{p_{1} M p_{1}} \times \mathrm{U}_{p_{3} M p_{3}} \times \mathrm{U}_{p_{2} M p_{2}}$. But the latter group is isomorphic to

$$
\left\{\left(\begin{array}{ccc}
u_{1} & 0 & 0 \\
0 & u_{3} & 0 \\
0 & 0 & u_{2}
\end{array}\right) \mid u_{j} \in \mathrm{U}_{p_{j} M p_{j}} \text { for } j=1,2,3\right\},
$$

which is a Lie subgroup of $\mathrm{U}_{M}$, hence $\mathrm{U}_{M^{\varphi}}=\mathrm{U}_{M^{\varphi_{1}}} \cap \mathrm{U}_{M^{\varphi_{2}}}$ is in turn a Lie subgroup of $\mathrm{U}_{M}$.

Corollary 2.9 For every $W^{*}$-algebra $M$ and $\varphi=\varphi^{*} \in M_{*}$, the coadjoint orbit of the Lie group $\mathrm{U}_{M}$ through $\varphi \in\left(\mathfrak{u}_{M}\right)_{*} \subseteq\left(\mathfrak{u}_{M}\right)^{*}$ has the structure of a $\mathrm{U}_{M}$-homogeneous weakly symplectic manifold which is weakly immersed into $\left(\mathfrak{u}_{M}\right)_{*}$. 
Proof. Just use Proposition 2.8 along with Theorem 7.3 in 28 .

Corollary 2.10 Let $M$ be an arbitrary $W^{*}$-algebra and consider the corresponding real Banach LiePoisson space $M_{*}^{s a}=\left\{\varphi \in M_{*} \mid \varphi=\varphi^{*}\right\}$. Then the characteristic distribution of $M_{*}^{\text {sa }}$ is integrable and all its maximal integral manifolds are symplectic leaves.

Proof. Use Corollary 2.9] along with Theorem 7.4 in 28] and note that all the coadjoint orbits referred to in Corollary [2.9] are connected since the unitary group of every $W^{*}$-algebra is connected.

Remark 2.11 It is noteworthy that the weakly symplectic manifolds given by Corollary 2.9 are sometimes strongly symplectic. For instance, this is the case of the coadjoint orbits of rank-one projections if we assume that $M=\mathcal{B}(\mathcal{H})$ for some complex Hilbert space $\mathcal{H}$ with the scalar product $(\cdot \mid \cdot)$.

In fact, for any $x \in \mathcal{H}$ with $\|x\|=1$ denote by $p_{x}=(\cdot \mid x) x$ the orthogonal projection of $\mathcal{H}$ onto the one-dimensional subspace $\mathbb{C} x$. Then $p_{x} \in M_{*}^{\text {sa }}$ and $u p_{x} u^{*}=p_{u x}$ for all unit vectors $x \in \mathcal{H}$ and all $u \in \mathrm{U}_{M}$. Thus, denoting by $S_{\mathcal{H}}$ the unit sphere of $\mathcal{H}$ (that is, the set of all unit vectors in $\mathcal{H}$ ) and by $\mathbb{P}(\mathcal{H}):=S_{\mathcal{H}} / \mathbb{T}$ the projective space of $\mathcal{H}$, it follows that the mapping

$$
S_{\mathcal{H}} \rightarrow M_{*}^{\mathrm{sa}}, \quad x \mapsto p_{x},
$$

induces a $\mathrm{U}_{M}$-equivariant diffeomorphism of $\mathbb{P}(\mathcal{H})$ onto the coadjoint orbit $\mathcal{P}_{1}:=\left\{p_{x} \mid x \in S_{\mathcal{H}}\right\}$. It is well known that the projective space $\mathbb{P}(\mathcal{H})$ is a strongly symplectic manifold (it is locally symplectomorphic to $\mathcal{H} / \mathbb{C} x_{0}$ with the symplectic form defined by the double of the imaginary part of the quotient scalar product, for an arbitrary unit vector $x_{0} \in \mathcal{H}$ ), hence our claim that $\mathcal{P}_{1}$ is strongly symplectic will follow as soon as we show that the aforementioned diffeomorphism $\mathcal{P}_{1} \simeq \mathbb{P}(\mathcal{H})$ is actually a symplectomorphism. To this end, fix a unit vector $x_{0} \in \mathcal{H}$. The symplectic structure of the coadjoint orbit $\mathcal{P}_{1}$ through $p_{x_{0}} \in M_{*}^{\mathrm{sa}}$ is defined by the skew symmetric bilinear form

$$
\omega_{x_{0}}: \mathfrak{u}_{M} \times \mathfrak{u}_{M} \rightarrow \mathbb{R}, \quad \omega_{x_{0}}\left(a_{1}, a_{2}\right)=i \operatorname{Tr}\left(p_{x_{0}}\left[a_{1}, a_{2}\right]\right)
$$

(see formula (7.5) in [28]). Since the elements of $\mathfrak{u}_{M}$ are skew-symmetric, it follows that for all $a_{1}, a_{2} \in$ $\mathfrak{u}_{M}$ we have

$$
\omega_{x_{0}}\left(a_{1}, a_{2}\right)=-i \cdot \operatorname{Tr}\left(\left(\cdot \mid\left[a_{1}, a_{2}\right] x_{0}\right) x_{0}\right)=i\left(\left[a_{1}, a_{2}\right] x_{0} \mid x_{0}\right)=2 \operatorname{Im}\left(a_{1} x_{0} \mid a_{2} x_{0}\right) .
$$

On the other hand, if we consider $\mathrm{U}_{M}^{x_{0}}=\left\{u \in \mathrm{U} \mid u x_{0} \in \mathbb{C} x_{0}\right\}$, which is the isotropy group of $\mathbb{C} x_{0} \in \mathbb{P}(\mathcal{H})$, then we have a $\mathrm{U}_{M}$-equivariant diffeomorphism $\mathrm{U}_{M} / \mathrm{U}_{M}^{x_{0}} \simeq \mathbb{P}(\mathcal{H})$, and the $\mathrm{U}_{M}$-invariant symplectic form of $\mathbb{P}(\mathcal{H})$ will be defined by the skew-symmetric bilinear form

$$
\omega_{x_{0}}^{\prime}: \mathfrak{u}_{M} \times \mathfrak{u}_{M} \rightarrow \mathbb{R}, \quad \omega_{x_{0}}\left(a_{1}, a_{2}\right)=2 \operatorname{Im}\left(a_{1} x_{0} \mid a_{2} x_{0}\right) .
$$

The above computation shows that $\omega_{x_{0}}=\omega_{x_{0}}^{\prime}$, and this concludes the proof of the fact that the $\mathrm{U}_{M}$-equivariant diffeomorphism $\mathcal{P}_{1} \simeq \mathbb{P}(\mathcal{H})$ is a symplectomorphism, whence the coadjoint orbit $\mathcal{P}_{1}$ is strongly symplectic. 
In the same special case when $M=\mathcal{B}(\mathcal{H})$ for some complex Hilbert space $\mathcal{H}$ (that is, when $M$ is a factor of type I), the result of Corollary 2.9] also follows by Corollary 7.7 in [28] along with Lemma 4.1 in [3. However, we conclude this section by showing that, in general, the previous Corollary [2.9] applies to coadjoint orbits that do not fall under the hypotheses of Corollary 7.7 in [28. To this end, we prove the following fact.

Proposition 2.12 Let $M$ be a von Neumann algebra on a complex Hilbert space $\mathcal{H}$. Assume that $M$ is $a \mathrm{II}_{1}$ factor with the faithful normal trace state $\tau$ and that there is a positive invertible element $h \in M$ with the spectral measure $E_{h}(\cdot)$ such that for some $v \in \mathcal{H} \backslash\{0\}$ the localized measure $\left\|E_{h}(\cdot) v\right\|^{2}$ has no atoms.

Now define

$$
\varphi: M \rightarrow \mathbb{C}, \quad \varphi(x)=\tau(h x) .
$$

Then $\varphi \in M_{*}$ is a faithful functional and there exists no family $\left\{e_{i}\right\}_{i \in I}$ of mutually orthogonal selfadjoint projections in $M$ satisfying $\sum_{i \in I} e_{i}=\mathbf{1}$ and $M^{\varphi}=\left\{\sum_{i \in I} e_{i} x e_{i} \mid x \in M\right\}$.

Proof. It is clear that $\varphi \in M_{*}$. Next, for every $x \in M$ we have

$$
\varphi\left(x^{*} x\right)=\tau\left(h x^{*} x\right)=\tau\left(x h x^{*}\right)=\tau\left(\left(h^{1 / 2} x^{*}\right)^{*}\left(h^{1 / 2} x^{*}\right)\right) .
$$

Since $\tau$ is faithful and $h$ is invertible, it then easily follows that $\varphi$ is faithful.

Now, to prove the property stated for $M^{\varphi}$, we first check that

$$
M^{\varphi}=\{a \in M \mid a h=h a\} .
$$

In fact, $a \in M^{\varphi}$ if and only if $\varphi(a x)=\varphi(x a)$ for all $x \in M$, that is, $\tau(h a x)=\tau(h x a)$ for $x \in M$. Since $\tau$ is a trace, the latter property is further equivalent to $\tau(h a x)=\tau(a h x)$ for all $x \in M$, hence to $h a=a h$.

Next let us assume that there exists a family $\left\{e_{i}\right\}_{i \in I}$ of self-adjoint projections in $M$ satisfying $e_{i} e_{j}=0$ whenever $i \neq j, \sum_{i \in I} e_{i}=1$ and $M^{\varphi}=\left\{\sum_{i \in I} e_{i} x e_{i} \mid x \in M\right\}$. According to the previous characterization of $M^{\varphi}$ we have that $h$ belongs to the center of $M^{\varphi}$. Then it follows at once that $e_{i} h e_{i}$ ( $\left.=e_{i} h=h e_{i}\right)$ belongs to the center of $e_{i} M e_{i}$ for each $i \in I$. On the other hand, since $M$ is a factor (i.e., its center reduces to the scalar multiples of the unit element) it follows by Corollary 3.15 in 32 that $e_{i} M e_{i}$ is in turn a factor, hence there exists $\lambda_{i} \in \mathbb{C}$ such that $e_{i} h=h e_{i}=\lambda_{i} e_{i}$, for arbitrary $i \in I$.

We now show that this fact contradicts the spectral assumption on $h$. In fact, since the measure $\left\|E_{h}(\cdot) v\right\|^{2}$ has no atoms, it follows that for each $i \in I$ we have $\left\|E_{h}\left(\left\{\lambda_{i}\right\}\right) v\right\|^{2}=0$, i.e., $E_{h}\left(\left\{\lambda_{i}\right\}\right) v=0$. On the other hand, since $e_{i} h=h e_{i}=\lambda_{i} e_{i}$, we get $e_{i} \leq E_{h}\left(\left\{\lambda_{i}\right\}\right)$, that is, $e_{i} E_{h}\left(\left\{\lambda_{i}\right\}\right)=E_{h}\left(\left\{\lambda_{i}\right\}\right) e_{i}=e_{i}$. Then $e_{i} v=e_{i} E_{h}\left(\left\{\lambda_{i}\right\}\right) v=0$ for every $i \in I$. Since $\sum_{i \in I} e_{i}=\mathbf{1}$, it then follows that $v=0$, a contradiction.

Example 2.13 A concrete situation where Proposition 2.12 applies is provided by Theorem 2.6.2 in 34. Specifically, let $M$ be the von Neumann algebra generated by the real parts $s(t):=\left(l(t)+l(t)^{*}\right) / 2$ of the left-creation operators $l(t)$ (for $t \in \mathcal{H}_{\mathbb{R}}$ ) on the full Fock space $\mathcal{T}\left(\mathcal{H}_{\mathbb{C}}\right.$ ) associated with the 
complexification $\mathcal{H}_{\mathbb{C}}$ of the real Hilbert space $\mathcal{H}_{\mathbb{R}}$ with $\operatorname{dim}\left(\mathcal{H}_{\mathbb{R}}\right)>1$. Then $M$ is a $\mathrm{II}_{1}$ factor with the trace defined by the vector form at the vacuum vector $v_{0}$.

Moreover, for arbitrary $t_{0} \in \mathcal{H}_{\mathbb{R}} \backslash\{0\}$, the operator $s\left(t_{0}\right)$ is self-adjoint and its spectral measure localized at the vacuum vector $v_{0}$ is absolutely continuous with respect to the Lebesgue measure, hence it has no atoms. As a matter of fact, the aforementioned localized spectral measure is given by the semicircle law

$$
\frac{2}{\pi\left\|t_{0}\right\|^{2}} \chi_{\left[-\left\|t_{0}\right\|,\left\|t_{0}\right\|\right]}(r) \sqrt{\left\|t_{0}\right\|^{2}-r^{2}} \mathrm{~d} r .
$$

Thus, for $\varepsilon>0$ arbitrary, $h:=\left\|t_{0}\right\|+\varepsilon+s\left(t_{0}\right) \in M$ is a positive invertible operator whose spectral measure localized at the vacuum vector $v_{0}$ (that is, the measure $\left.\left\|E_{h}(\cdot) v_{0}\right\|^{2}\right)$ is absolutely continuous with respect to the Lebesgue measure on $\mathbb{R}$.

\section{Orbits of adjoint actions}

Let $\mathcal{B}(\mathcal{H})$ denote the space of all bounded operators and $\mathrm{U}_{\mathcal{B}(\mathcal{H})}$ the group of all unitary operators on the complex Hilbert space $\mathcal{H}$.

Theorem 3.1 ([12]) Let $\mathcal{H}$ be a complex Hilbert space, $T \in \mathcal{B}(\mathcal{H}), \mathrm{U}_{\mathcal{B}(\mathcal{H})}(T)$ the unitary orbit through $T$, and

$$
\alpha: \mathrm{U}_{\mathcal{B}(\mathcal{H})} \rightarrow \mathrm{U}_{\mathcal{B}(\mathcal{H})}(T)(\subseteq \mathcal{B}(\mathcal{H})), \quad V \mapsto V T V^{*}
$$

the corresponding orbit map. The following assertions are equivalent:

(i) The map $\alpha$ has local continuous cross sections if the unitary orbit $\mathrm{U}_{\mathcal{B}(\mathcal{H})}(T)$ is endowed with the relative topology inherited from $\mathcal{B}(\mathcal{H})$.

(ii) The unitary orbit $\mathrm{U}_{\mathcal{B}(\mathcal{H})}(T)$ is closed in $\mathcal{B}(\mathcal{H})$.

(iii) The sub-C ${ }^{*}$-algebra generated by $T$ in $\mathcal{B}(\mathcal{H})$ is finite dimensional.

(iv) There exist operators $A$ and $B$ on certain finite dimensional spaces such that $T$ is unitarily equivalent to the Hilbert space operator defined by the infinite block-diagonal matrix

$$
\left(\begin{array}{cccccc}
A & & & & & 0 \\
& B & & & & \\
& & B & & & \\
& & & \ddots & & \\
& & & & B & \\
0 & & & & & \ddots
\end{array}\right) .
$$

(v) The unitary orbit $\mathrm{U}_{\mathcal{B}(\mathcal{H})}(T)$ is a smooth submanifold of $\mathcal{B}(\mathcal{H})$. 
Proof. See Theorem 1.1 in [12] or Theorem 4.1 in [6] for the fact that assertions (i)-(iv) are equivalent. Moreover, these assertions are equivalent to (v) by the results of [1]; see Theorems 1.1 and 1.3 in [2].

Concerning Theorem 3.1(i), we note that the existence of global cross sections of the orbit map was investigated in [30. In fact, according to Theorems 4 and 7 in 30, and using the notation of Theorem [3.1] a global continuous cross section of $\alpha$ can be constructed if and only if we can choose $A=B$ in Theorem 3.1 (iv).

Theorem $3.2([5])$ Let $\mathcal{H}$ be a complex Hilbert space, $T \in \mathcal{B}(\mathcal{H})$, and define

$$
\operatorname{ad} T: \mathcal{B}(\mathcal{H}) \rightarrow \mathcal{B}(\mathcal{H}) \quad \text { by } \quad A \mapsto[T, A] .
$$

Furthermore denote by $\mathfrak{S}_{1}$ the ideal of trace class operators on $\mathcal{H}$. Then the following assertions are equivalent:

(i) The operator ad $T$ has closed range in $\mathcal{B}(\mathcal{H})$.

(ii) For every complex polynomial $p$ the operator $p(T)$ has closed range in $\mathcal{H}$ and there exists a non-zero polynomial $p_{0}$ such that $p_{0}(T)=0$.

(iii) The operator $T$ is similar to an operator that generates a finite-dimensional sub-C ${ }^{*}$-algebra of $\mathcal{B}(\mathcal{H})$.

(iv) The operator $\left.\operatorname{ad} T\right|_{\mathfrak{S}_{1}}$ has closed range in $\mathfrak{S}_{1}$.

Proof. The fact that the assertions (i)-(iii) are equivalent can be found in [5].

The fact that (i) is equivalent to (iv) is also well known and follows by an easy duality argument (see e.g., Theorem 3.5(ii) and the proof of Proposition 3.12 in [14). Thus, first recall that the range of a Banach space operator is closed if and only if the range of its dual operator is closed. Since the Banach space dual to $\mathfrak{S}_{1}$ is $\mathcal{B}(\mathcal{H})$ and the operator dual to $\left.\operatorname{ad} T\right|_{\mathfrak{S}_{1}}$ is $-\operatorname{ad} T^{\prime}$ (where $T^{\prime} \in \mathcal{B}(\mathcal{H}$ ) is the operator dual to $T$ ), while $T^{\prime}$ is conjugate-similar to $T^{*}$, it then follows that the range of $\left.\operatorname{ad} T\right|_{\mathfrak{S}_{1}}$ is closed if and only if the range of ad $T^{*}$ is closed. Furthermore, the range of ad $T^{*}$ is closed if and only if the range of $\operatorname{ad} T$ is closed, as a consequence of the fact that (i) $\Leftrightarrow$ (ii).

Remark 3.3 More details on Theorem 3.2 can be found in Chapter 15 of the book $[\underline{6}$.

Proposition 3.4 If $T \in \mathfrak{u}_{\mathcal{B}(\mathcal{H})}$ and we denote

$$
\mathrm{U}_{\mathcal{B}(\mathcal{H}), T}=\left\{U \in \mathrm{U}_{\mathcal{B}(\mathcal{H})} \mid U T U^{-1}=T\right\},
$$

then $\mathrm{U}_{\mathcal{B}(\mathcal{H}), T}$ is a Lie subgroup of $\mathrm{U}_{\mathcal{B}(\mathcal{H})}$.

Proof. It follows by Theorem 8.12 in [7] that $\mathrm{U}_{\mathcal{B}(\mathcal{H}), T}$ is a Banach Lie group with respect to the topology inherited from $U_{\mathcal{B}(\mathcal{H})}$ and with the Lie algebra $\mathfrak{u}_{\mathcal{B}(\mathcal{H}), T}:=\operatorname{Ker}\left(\operatorname{ad}_{\mathfrak{u}_{\mathcal{B}(\mathcal{H})}} T\right)$. 
So it only remains to check that $\mathfrak{u}_{\mathcal{B}(\mathcal{H}), T}$ is a split subspace of $\mathfrak{u}_{\mathcal{B}(\mathcal{H})}$, which is well-known. Just pick an invariant mean $\operatorname{LIM}_{\alpha \rightarrow \infty}$ on the (Abelian, hence amenable) group $(\mathbb{R},+$ ), and define a continuous linear map

$$
E: \mathfrak{u}_{\mathcal{B}(\mathcal{H})} \rightarrow \mathfrak{u}_{\mathcal{B}(\mathcal{H})} \text { with } E^{2}=E \text { and } \operatorname{Ran} E=\operatorname{Ker}\left(\operatorname{ad}_{\mathfrak{u}_{\mathcal{B}(\mathcal{H})}} T\right)
$$

in the following way: for all $S \in \mathfrak{u}_{\mathcal{B}(\mathcal{H})}$ and $f, g \in \mathcal{H}$ let

$$
(E(S) f \mid g)=\operatorname{LIM}_{\alpha \rightarrow \infty}(S(\exp (\alpha T)) f \mid(\exp (\alpha T)) g) .
$$

We recall that $\underset{\alpha \rightarrow \infty}{\operatorname{LIM}}$ is just a suggestive notation for a positive linear functional $m: \ell^{\infty}(\mathbb{R}, \mathbb{C}) \rightarrow \mathbb{C}$ satisfying $\|m\|=1$ and

$$
\left(\forall \xi \in \ell^{\infty}(\mathbb{R}, \mathbb{C})\right)(\forall \alpha \in \mathbb{R}) \quad m(\xi)=m\left(\xi_{\alpha}\right),
$$

where $\ell^{\infty}(\mathbb{R}, \mathbb{C})$ is the commutative $C^{*}$-algebra of all bounded functions $\xi: \mathbb{R} \rightarrow \mathbb{C}$, and $\xi_{\alpha}(\beta):=\xi(\alpha+\beta)$ whenever $\xi \in \ell^{\infty}(\mathbb{R}, \mathbb{C})$ and $\alpha, \beta \in \mathbb{R}$. The existence of a functional $m$ with the aforementioned properties follows by Theorem 1.2.1 in [16], and our notation $\underset{\alpha \rightarrow \infty}{\operatorname{LIM}}$ is then introduced by

$$
\left(\forall \xi \in \ell^{\infty}(\mathbb{R}, \mathbb{C})\right) \quad \operatorname{LIM}_{\alpha \rightarrow \infty} \xi(\alpha):=m(\xi) .
$$

Now the fact that the map $E: \mathfrak{u}_{\mathcal{B}(\mathcal{H})} \rightarrow \mathfrak{u}_{\mathcal{B}(\mathcal{H})}$ has the properties claimed above follows by Theorem 16(b) in 21] applied for the unitary representation $\alpha \mapsto \exp (\alpha T)$ of the Abelian group $(\mathbb{R},+)$.

\section{Symplectic leaves in preduals of operator ideals}

In this section and in the following one, $\mathcal{H}$ stands for a separable complex Hilbert space, and GL $(\mathcal{H})$ for the set of all invertible bounded linear operators on $\mathcal{H}$.

Definition 4.1 Let $\mathcal{H}$ be a complex Hilbert space and $\mathfrak{F}$ the ideal of all finite-rank operators on $\mathcal{H}$. For every two-sided ideal $\mathfrak{I}$ of $\mathcal{B}(\mathcal{H})$ we shall use the following notation:

$$
\begin{aligned}
\mathrm{U}_{\mathfrak{I}} & =\mathrm{U}_{\mathcal{B}(\mathcal{H})} \cap(\mathbf{1}+\mathfrak{I}) \\
\mathfrak{u}_{\mathfrak{I}} & =\mathfrak{u}_{\mathcal{B}(\mathcal{H})} \cap \mathfrak{I} .
\end{aligned}
$$

For later use, we now recall a few facts concerning Banach ideals of operators on the complex Hilbert space $\mathcal{H}$ (see [15] and also [13]).

Remark 4.2 (i) By Banach ideal we mean a two-sided ideal $\mathfrak{I}$ of $\mathcal{B}(\mathcal{H})$ equipped with a norm $\|\cdot\|_{\mathfrak{I}}$ satisfying $\|T\| \leq\|T\|_{\mathfrak{I}}=\left\|T^{*}\right\|_{\mathfrak{I}}$ and $\|A T B\|_{\mathfrak{I}} \leq\|A\|\|T\|_{\mathfrak{I}}\|B\|$ whenever $A, B \in \mathcal{B}(\mathcal{H})$.

(ii) Let $\widehat{c}$ be the vector space of all sequences of real numbers $\left\{\xi_{j}\right\}_{j \geq 1}$ such that $\xi_{j}=0$ for all but finitely many indices. A symmetric norming function is a function $\Phi: \widehat{c} \rightarrow \mathbb{R}$ satisfying the following conditions:

I) $\Phi(\xi)>0$ whenever $0 \neq \xi \in \widehat{c}$, 
II) $\Phi(\alpha \xi)=|\alpha| \Phi(\xi)$ whenever $\alpha \in \mathbb{R}$ and $\xi \in \widehat{c}$,

III) $\Phi(\xi+\eta) \leq \Phi(\xi)+\Phi(\eta)$ whenever $\xi, \eta \in \widehat{c}$,

IV) $\Phi((1,0,0, \ldots))=1$,

V) $\Phi\left(\left\{\xi_{j}\right\}_{j \geq 1}\right)=\Phi\left(\left\{\xi_{\pi(j)}\right\}_{j \geq 1}\right)$ whenever $\left\{\xi_{j}\right\}_{j \geq 1} \in \widehat{c}$ and $\pi:\{1,2, \ldots\} \rightarrow\{1,2, \ldots\}$ is bijective.

Any symmetric norming function $\Phi$ gives rise to two Banach ideals $\mathfrak{S}_{\Phi}$ and $\mathfrak{S}_{\Phi}^{(0)}$ as follows. For every bounded sequence of real numbers $\xi=\left\{\xi_{j}\right\}_{j \geq 1}$ define

$$
\Phi(\xi):=\sup _{n \geq 1} \Phi\left(\xi_{1}, \xi_{2}, \ldots, \xi_{n}, 0,0, \ldots\right) \in[0, \infty] .
$$

For all $T \in \mathcal{B}(\mathcal{H})$ denote

$$
\|T\|_{\Phi}:=\Phi\left(\left\{s_{j}(T)\right\}_{j \geq 1}\right) \in[0, \infty],
$$

where $s_{j}(T)=\inf \{\|T-F\| \mid F \in \mathcal{B}(\mathcal{H}), \operatorname{rank} F<j\}$ whenever $j \geq 1$. With this notation we can define

$$
\begin{aligned}
\mathfrak{S}_{\Phi} & =\left\{T \in \mathcal{B}(\mathcal{H}) \mid\|T\|_{\Phi}<\infty\right\}, \\
\mathfrak{S}_{\Phi}^{(0)} & =\overline{\mathfrak{F}}^{\|\cdot\|_{\Phi}} \quad\left(\subseteq \mathfrak{S}_{\Phi}\right),
\end{aligned}
$$

that is, $\mathfrak{S}_{\Phi}^{(0)}$ is the $\|\cdot\|_{\Phi}$-closure of the finite-rank operators $\mathfrak{F}$ in $\mathfrak{S}_{\Phi}$. Then $\|\cdot\|_{\Phi}$ is a norm making $\mathfrak{S}_{\Phi}$ and $\mathfrak{S}_{\Phi}^{(0)}$ into Banach ideals (see $\S 4$ in Chapter III in [15]). Actually, every separable Banach ideal equals $\mathfrak{S}_{\Phi}^{(0)}$ for some symmetric norming function $\Phi$ (see Theorem 6.2 in Chapter III in [15]).

(iii) For every symmetric norming function $\Phi: \widehat{c} \rightarrow \mathbb{R}$ there exists a unique symmetric norming function $\Phi^{*}: \widehat{c} \rightarrow \mathbb{R}$ such that

$$
\Phi^{*}(\eta)=\sup \left\{\frac{1}{\Phi(\xi)} \sum_{j=1}^{\infty} \xi_{j} \eta_{j} \mid \xi=\left\{\xi_{j}\right\}_{j \geq 1} \in \widehat{c} \text { and } \xi_{1} \geq \xi_{2} \geq \cdots \geq 0\right\}
$$

whenever $\eta=\left\{\eta_{j}\right\}_{j \geq 1} \in \widehat{c}$ and $\eta_{1} \geq \eta_{2} \geq \cdots \geq 0$. The function $\Phi^{*}$ is said to be adjoint to $\Phi$ and we always have $\left(\Phi^{*}\right)^{*}=\Phi$ (see Theorem 11.1 in Chapter III in [15]). For instance, if $1 \leq p, q \leq \infty$, $1 / p+1 / q=1, \Phi_{p}(\xi)=\|\xi\|_{\ell^{p}}$ and $\Phi_{q}(\xi)=\|\xi\|_{\ell q}$ whenever $\xi \in \widehat{c}$, then $\left(\Phi_{p}\right)^{*}=\Phi_{q}$. If $\Phi$ is any symmetric norming function then the topological dual of the Banach space $\mathfrak{S}_{\Phi}^{(0)}$ is isometrically isomorphic to $\mathfrak{S}_{\Phi^{*}}$ by means of the duality pairing

$$
\mathfrak{S}_{\Phi^{*}} \times \mathfrak{S}_{\Phi}^{(0)} \rightarrow \mathbb{C}, \quad(T, S) \mapsto \operatorname{Tr}(T S)
$$

(see Theorems 12.2 and 12.4 in Chapter III in [15]).

Lemma 4.3 Let $k$ be a positive integer and

$$
\mathfrak{F}_{k}:=\{T \in \mathfrak{F} \mid \operatorname{rank} T \leq k\} .
$$

Then for every symmetric norming function $\Phi$ the norms $\|\cdot\|_{\Phi}$ and $\|\cdot\|$ define the same topology on the set $\mathfrak{F}_{k}$. 
Proof. We essentially follow the idea of proof of Lemma 2.1 in 9]. Inequalities (3.12) in Chapter III in [15] show that

$$
\xi_{1}=\Phi_{\infty}(\xi) \leq \Phi(\xi) \leq \Phi_{1}(\xi)=\sum_{j=1}^{\infty} \xi_{j}
$$

whenever $\xi=\left\{\xi_{j}\right\}_{j \geq 1} \in \widehat{c}$ and $\xi_{1} \geq \xi_{2} \geq \cdots \geq 0$. Since for each $F \in \mathfrak{F}_{2 k}$ we have $s_{2 k+1}(F)=$ $s_{2 k+2}(F)=\cdots=0$, we get

$$
\left(\forall F \in \mathfrak{F}_{2 k}\right) \quad\|F\|=\|F\|_{\Phi_{\infty}} \leq\|F\|_{\Phi} \leq\|F\|_{\Phi_{1}}=\sum_{j=1}^{\infty} s_{j}(F) \leq 2 k \cdot s_{1}(F)=2 k\|F\| .
$$

On the other hand, the difference of any two operators in $\mathfrak{F}_{k}$ clearly belongs to $\mathfrak{F}_{2 k}$, so that

$$
\left(\forall F_{1}, F_{2} \in \mathfrak{F}_{k}\right) \quad\left\|F_{1}-F_{2}\right\| \leq\left\|F_{1}-F_{2}\right\|_{\Phi} \leq 2 k\left\|F_{1}-F_{2}\right\|,
$$

and the proof is complete.

Lemma 4.4 Let $\mathcal{X}_{0}$ be a reflexive real Banach space and $A_{0}: \mathcal{X}_{0} \rightarrow \mathcal{X}_{0}$ a bounded linear operator such that $\sup _{t \in \mathbb{R}}\left\|\exp \left(t A_{0}\right)\right\|<\infty$. Then $\mathcal{X}_{0}=\operatorname{Ker} A_{0} \oplus \overline{\operatorname{Ran} A_{0}}$.

Proof. First endow the complexified space $\mathcal{X}:=\mathcal{X}_{0} \oplus$ i $\mathcal{X}_{0}$ with a norm making the conjugation

$$
C: \mathcal{X} \rightarrow \mathcal{X}, \quad x+\mathrm{i} y \mapsto x-\mathrm{i} y,
$$

into an isometry (see e.g., Notation 1.1 in [7] for a method to define such a norm). Thus for all $x, y \in \mathcal{X}_{0}$ we have

$$
\|x\| \leq \frac{\|x+\mathrm{i} y\|+\|x-\mathrm{i} y\|}{2}=\|x+\mathrm{i} y\| .
$$

Then denote by $A \in \mathcal{B}(\mathcal{X})$ the unique complex-linear operator whose restriction to $\mathcal{X}_{0}$ is $A_{0}$ and commutes with the conjugation, that is, $A C=C A$.

On the other hand, denote $M:=\sup _{t \in \mathbb{R}}\left\|\exp \left(t A_{0}\right)\right\|$. Then for all $z=x+\mathrm{i} y \in \mathcal{X}$ and $t \in \mathbb{R}$ we have

$$
\|\exp (t A) z\|=\left\|\exp \left(t A_{0}\right) x+\mathrm{i} \exp \left(t A_{0}\right) y\right\| \leq\|x\|+\|y\| \leq 2 M\|z\|,
$$

where the last inequality follows by (4.1). Thus $\sup _{t \in \mathbb{R}}\|\exp (t A)\| \leq 2 M$. Then it is well known that the norm defined on $\mathcal{X}$ by $\|z\|_{1}:=\sup _{t \in \mathbb{R}}\|\exp (t A) z\|$ is equivalent to $\|\cdot\|$ and has the property that $\|\exp (t A)\|_{1}=1$ for all $t \in \mathbb{R}$ (see e.g., Lemma 7 in $\S 2$ in $[10$ ). Since the Banach space $\mathcal{X}$ is reflexive, it then follows by Corollary 4.5 in 23 that $\mathcal{X}=\operatorname{Ker}(\mathrm{i} A) \oplus \overline{\operatorname{Ran}(\mathrm{i} A)}$, that is, $\mathcal{X}=\operatorname{Ker} A \oplus \overline{\operatorname{Ran} A}$.

Now, we have $C A=A C, \mathcal{X}_{0}=\{z \in \mathcal{X} \mid C(z)=z\}$ and $\left.A\right|_{\mathcal{X}_{0}}=A_{0}$, so it is straightforward to show that $\mathfrak{X}_{0}=\operatorname{Ker} A_{0} \oplus \overline{\operatorname{Ran} A_{0}}$. 
Proposition 4.5 Let $\mathfrak{I}$ be a Banach ideal whose underlying Banach space is reflexive, $T \in \mathfrak{u}_{\mathfrak{I}}$, and denote

$$
\mathrm{U}_{\mathfrak{J}, T}=\left\{U \in \mathrm{U}_{\mathfrak{J}} \mid U T U^{-1}=T\right\} .
$$

Then $\mathrm{U}_{\mathfrak{J}, T}$ is a Lie subgroup of $\mathrm{U}_{\mathfrak{J}}$.

Proof. We first recall from Proposition 10.11 in [7] that $\mathrm{U}_{\mathfrak{J}}$ is real Banach Lie group whose Banach Lie algebra is $\mathfrak{u}_{\mathfrak{J}}$ and that the inclusion map $\mathrm{U}_{\mathfrak{J}} \hookrightarrow \mathrm{U}_{\mathcal{B}(\mathcal{H})}$ is a homomorphism of Banach Lie groups (see also Lemma 5.1 below). Since $\mathrm{U}_{\mathfrak{J}, T}$ is just the inverse image of $\mathrm{U}_{\mathcal{B}(\mathcal{H}), T}$ by the aforementioned inclusion map, it follows from Proposition 3.4 and Lemma IV.11 in [24] that $\mathrm{U}_{\mathfrak{J}, T}$ is a Banach Lie group with respect to the topology inherited from $U_{\mathfrak{J}}$ and whose Lie algebra is $\mathfrak{u}_{\mathfrak{J}, T}=\operatorname{Ker}\left(\operatorname{ad}_{\mathfrak{u}_{\mathfrak{J}}} T\right)$.

It only remains to be shown that $\mathfrak{u}_{\mathfrak{J}, T}$ is a split subspace of $\mathfrak{u}_{\mathfrak{J}}$. But this follows by Lemma 4.4 since for all $t \in \mathbb{R}$ and $S \in \mathfrak{u}_{\mathfrak{J}}$ we have $\left(\exp \left(\operatorname{ad}_{\mathfrak{u}_{\mathfrak{v}}} t T\right)\right) S=\mathrm{e}^{t T} S \mathrm{e}^{-t T}$, whence $\left\|\exp \left(\operatorname{ad}_{\mathfrak{u}_{\mathfrak{J}}} t T\right)\right\| \leq 1$.

Corollary 4.6 Let $(\mathfrak{B}, \mathfrak{J})$ be a pair of Banach ideals whose underlying Banach spaces are reflexive and assume that the trace pairing

$$
\mathfrak{B} \times \mathfrak{J} \rightarrow \mathbb{C}, \quad(T, S) \mapsto \operatorname{Tr}(T S)
$$

is well defined and induces a topological isomorphism of the topological dual $\mathfrak{B}^{*}$ onto $\mathfrak{J}$. Then the characteristic distribution of the real Banach Lie-Poisson space $\mathfrak{u}_{\mathfrak{B}}=\left(\mathfrak{u}_{\mathfrak{J}}\right)_{*}$ is integrable and all its maximal integral manifolds are symplectic leaves.

Proof. The proof is similar to that of Corollaries [2.9] and [2.10] using Proposition 4.5] instead of Proposition 2.8

Example 4.7 An obvious example of a pair of Banach ideals $(\mathfrak{B}, \mathfrak{J})$ to which Corollary 4.6 applies is a pair of Schatten ideals $\left(\mathfrak{S}_{p}, \mathfrak{S}_{q}\right)$ with $p, q \in(1, \infty)$ and $1 / p+1 / q=1$. More sophisticated pairs of Banach ideals in duality arise in the duality theory of operator ideals; see Remark 4.2(iii).

We now consider the problem of constructing invariant complex structures compatible with the symplectic structures on certain of the leaves in Corollary 4.6. This problem can be treated by the techniques used in the proof of Theorem VII.6 in 24.

Proposition 4.8 Assume that the pair of Banach ideals $(\mathfrak{B}, \mathfrak{J})$ has the properties that the Banach Lie group $\mathrm{U}_{\mathfrak{J}}$ is connected and the trace pairing

$$
\mathfrak{B} \times \mathfrak{J} \rightarrow \mathbb{C}, \quad(T, S) \mapsto \operatorname{Tr}(T S)
$$

is well defined and induces a topological isomorphism of the topological dual $\mathfrak{B}^{*}$ onto $\mathfrak{J}$. Let $T \in \mathfrak{u}_{\mathfrak{B}} \cap \mathfrak{F}$ be a given element and denote

$$
\mathrm{U}_{\mathfrak{J}, T}=\left\{U \in \mathrm{U}_{\mathfrak{J}} \mid U T U^{-1}=T\right\} .
$$

Then the homogeneous space $\mathrm{U}_{\mathfrak{J}} / \mathrm{U}_{\mathfrak{J}, T}$ has a $\mathrm{U}_{\mathfrak{J}}$-invariant weakly Kähler structure and this homogeneous space is weakly immersed into $\mathfrak{u}_{\mathfrak{B}}$. 
Proof. $\quad 1^{\circ}$ Preparations: Denote $\sigma(T)=\left\{\lambda_{0}, \lambda_{1}, \ldots, \lambda_{n}\right\}$ with $\lambda_{0}=0$, and $\mathcal{H}_{i}=\operatorname{Ker}\left(T-\lambda_{i} \mathbf{1}\right)$ for $i=0, \ldots, n$. Since $T^{*}=-T$, it follows that we have the orthogonal direct sum

$$
\mathcal{H}=\mathcal{H}_{1} \oplus \cdots \oplus \mathcal{H}_{n} \oplus \mathcal{H}_{0}
$$

Moreover, $\operatorname{dim} \mathcal{H}_{i}<\infty$ for $i=1, \ldots, n$, since $T \in \mathfrak{F}$.

Henceforth we will think of the operators on $\mathcal{H}$ as operator matrices with respect to the above orthogonal decomposition. In particular we have

$$
T=\left(\begin{array}{cccc}
\lambda_{1} & & & 0 \\
& \ddots & & \\
& & \lambda_{n} & \\
0 & & & \mathbf{0}
\end{array}\right),
$$

which easily implies that

$$
\sigma\left(\left.\operatorname{ad} T\right|_{\mathfrak{J}}\right)=\left\{\lambda_{i}-\lambda_{j} \mid 0 \leq i, j \leq n\right\}
$$

and that

$$
\mathfrak{J}=\bigoplus_{\mu \in \sigma\left(\left.\operatorname{ad} T\right|_{\mathfrak{J}}\right)} \operatorname{Ker}\left(\left.\operatorname{ad} T\right|_{\mathfrak{J}}-\mu\right)
$$

$2^{\circ}$ The isotropy group: In particular, it follows that $\operatorname{Ker}\left(\left.\operatorname{ad} T\right|_{\mathfrak{J}}\right)$ is complemented in $\mathfrak{J}$, hence the Lie algebra $\mathfrak{u}_{\mathfrak{J}, T}=\operatorname{Ker}\left(\left.\operatorname{ad} T\right|_{\mathfrak{u}_{\mathfrak{J}}}\right)$ of the Lie group $\mathrm{U}_{\mathfrak{J}, T}$ is complemented in $\mathfrak{u}_{\mathfrak{J}}$. Since $\mathrm{U}_{\mathfrak{J}, T}$ is a Lie group with the topology inherited from $\mathrm{U}_{\mathfrak{J}}$ (which follows as in the first part of the proof of Proposition 4.5), we see that $\mathrm{U}_{\mathfrak{J}, T}$ is in fact a Lie subgroup of $\mathrm{U}_{\mathfrak{J}}$.

$3^{\circ}$ The complex structure: Since $T^{*}=-T$, it follows that $\sigma(T) \subseteq i \mathbb{R}$. Now we can apply Proposition 8.7 in [7] with $S=\mathrm{i}[0, \infty), \mathfrak{z}=\mathbb{R}$ and $\Psi(\gamma)=\gamma\left(\left.\operatorname{ad} T\right|_{\mathfrak{u}_{\mathfrak{\gamma}}}\right)$ for $\gamma \in \mathbb{R}$ to deduce that the subspace

$$
\mathfrak{p}:=\bigoplus_{\mu \in(-S) \cap \sigma\left(\left.\operatorname{ad} T\right|_{\mathfrak{J}}\right)} \operatorname{Ker}\left(\left.\operatorname{ad} T\right|_{\mathfrak{J}}-\mu\right)
$$

of $\mathfrak{J}$ has the properties

(i) $\left[\mathfrak{u}_{\mathfrak{J}, T}, \mathfrak{p}\right] \subseteq \mathfrak{p}$,

(ii) $\mathfrak{p} \cap \overline{\mathfrak{p}}=\mathfrak{u}_{\mathfrak{J}, T}+\mathfrak{i u}_{\mathfrak{J}, T}$,

(iii) $\mathfrak{p}+\overline{\mathfrak{p}}=\mathfrak{J}$, and

(iv) $\mathfrak{p}$ is complemented in $\mathfrak{J}$.

Actually it is clear from the expression of $\mathfrak{p}$ that we have

(i') $V \mathfrak{p} V^{-1} \subseteq \mathfrak{p}$ if $V \in \mathrm{U}_{\mathfrak{J}, T}$, 
hence Theorem 8.4 in [7] shows that there exists a $U_{\mathfrak{J}}$-invariant complex structure on the homogeneous space $\mathrm{U}_{\mathfrak{J}} / \mathrm{U}_{\mathfrak{J}, T}$.

$4^{\circ}$ The symplectic structure: Now consider the continuous 2-cocycle of $\mathfrak{u}_{\mathfrak{J}}$ (actually 2-coboundary) defined by $T \in\left(\mathfrak{u}_{\mathfrak{J}}\right)_{*} \subseteq \mathfrak{u}_{\mathfrak{J}}^{*}$ :

$$
\omega_{T}: \mathfrak{u}_{\mathfrak{J}} \times \mathfrak{u}_{\mathfrak{J}} \rightarrow \mathbb{R}, \quad \omega_{T}(X, Y)=\operatorname{Tr}(T[X, Y]) .
$$

This is just the 2-cocycle that gives rise to the $\mathrm{U}_{\mathfrak{J}}$-invariant weakly symplectic structure of $\mathrm{U}_{\mathfrak{J}} / \mathrm{U}_{\mathfrak{J}, T}$ constructed in Theorem 7.3 in [28].

$5^{\circ}$ Kähler compatibility: Note that the above expression of $\mathfrak{p}$ (lower triangular block matrices, provided we arrange increasingly the eigenvalues of $T$ on $i \mathbb{R})$ immediately shows that we have $\omega_{T}(\mathfrak{p} \times \mathfrak{p})=$ $\{0\}$, that is, $\mathfrak{p}$ is actually a complex polarization of $\mathfrak{u}_{\mathfrak{J}}$ relative to the continuous 2-cocycle $\omega_{T}$ (see e.g., Definition 9.10 in [7]). Furthermore, note that

$$
\mathfrak{u}_{\mathfrak{J}, T}=\operatorname{Ker}\left(\left.\operatorname{ad} T\right|_{\mathfrak{u}_{\mathfrak{J}}}\right)=\left\{X \in \mathfrak{u}_{\mathfrak{J}} \mid\left(\forall Y \in \mathfrak{u}_{\mathfrak{J}}\right) \quad \omega_{T}(X, Y)=0\right\} .
$$

Now a standard reasoning (see e.g., page 77 in 24]) shows that the complex and weakly symplectic invariant structures on the homogeneous space $\mathrm{U}_{\mathfrak{J}} / \mathrm{U}_{\mathfrak{J}, T}$ are compatible, thus making it into a weakly pseudo-Kähler manifold. This manifold is actually Kähler since for all $Z \in \mathfrak{p}$ we have $-\mathrm{i} \omega_{T}\left(Z, Z^{*}\right) \geq 0$ just as in the proof of Lemma VII.4 in [24].

Remark 4.9 In connection with the hypothesis of Proposition 4.8 we note that if $\mathfrak{J}=\mathcal{B}(\mathcal{H})$ then $\mathrm{U}_{\mathfrak{J}}=\mathrm{U}_{\mathcal{B}(\mathcal{H})}$ is well known to be connected. Also, if $\mathfrak{J}$ is a separable Banach ideal, then the Banach Lie group $\mathrm{U}_{\mathfrak{J}}$ is connected as an easy consequence of Theorem (B) in [29] and Lemma [5.1] below. In fact, Theorem (B) in [29] implies that, for a separable Banach ideal $\mathfrak{J}$, the Banach Lie group $\mathrm{GL}_{\mathfrak{J}}$ has the same homotopy groups as the direct limit group $\operatorname{GL}(\infty, \mathbb{C})=\lim \operatorname{GL}(n, \mathbb{C})$, with respect to the natural embeddings $\mathrm{GL}(n, \mathbb{C}) \hookrightarrow \mathrm{GL}(n+1, \mathbb{C})$,

$$
A \mapsto\left(\begin{array}{cc}
A & 0 \\
0 & 1
\end{array}\right)
$$

In particular, $\mathrm{GL}_{\mathfrak{J}}$ is connected. Then Lemma 5.1 below easily implies that $\mathrm{U}_{\mathfrak{J}}$ is connected.

Thus, in the special case when $\mathfrak{J}$ is separable and $\mathfrak{B} \subseteq \mathfrak{J}$, the conclusion of the above Proposition 4.8 also follows by the results in Chapter 10 in [7].

Remark 4.10 We mention that in the special case when in Proposition 4.8 we have $\mathfrak{B}=\mathfrak{F}=\mathfrak{S}_{2}$ (the Hilbert-Schmidt ideal) the homogeneous space $\mathrm{U}_{\mathfrak{J}} / \mathrm{U}_{\mathfrak{J}, T}$ is always a strongly Kähler manifold; see [24] for details.

\section{$5 \quad$ Embedded orbits in operator ideals}

The unitary orbits of finite-rank self-adjoint operators are embedded submanifolds of $\mathcal{B}(\mathcal{H})$, according to the results of Andruchow and Stojanoff [1], 2] (see Theorem 3.1] above). In this section we prove 
a more general version of a similar result of Bona [8, 9] saying that, on unitary orbits of finite-rank operators on Hilbert spaces, the natural quotient topology coincides with the trace-class topology. This fact actually follows by Theorem 3.1 above and an easy topological remark (see Lemma 5.9 below), so that a version of Theorem 3.1 involving operator ideals will automatically lead to a generalization of the aforementioned result in [8, 9]. That generalization will concern smaller unitary orbits consisting in operators of the form $V^{*} T V$, where $V$ runs through the set of all unitary operators belonging to $\mathbf{1}+\mathfrak{I}$, for a suitable operator ideal $\mathfrak{I}$. Additionally, we provide conditions ensuring the existence of invariant Kähler structures on these smaller unitary orbits (Theorem 5.10).

We now prepare to establish a version of Theorem 3.1 (iii) $\Rightarrow$ (i)) in the more general setting of operator ideals (see Theorem 5.3 below). The key idea consists in showing that the main steps of the proof of Theorem 2.1 in [12 can be carried out in the present setting.

Lemma 5.1 Let $\mathfrak{I}$ be a Banach ideal of $\mathcal{B}(\mathcal{H})$. Then $\mathrm{GL}_{\mathfrak{I}}:=\mathrm{GL}(\mathcal{H}) \cap(1+\mathfrak{I})$ is a complex Banach Lie group, $\mathrm{U}_{\mathfrak{I}}:=\mathrm{U}_{\mathcal{B}(\mathcal{H})} \cap(\mathbf{1}+\mathfrak{I})$ is a real Lie subgroup of $\mathrm{GL}_{\mathfrak{I}}, \mathfrak{p}_{\mathfrak{I}}:=\left\{A \in \mathfrak{I} \mid A=A^{*}\right\}$ is a real Banach space with the norm inherited from $\mathfrak{I}$, and the map

$$
\Phi: \mathrm{U}_{\mathfrak{I}} \times \mathfrak{p}_{\mathfrak{I}} \rightarrow \mathrm{GL}_{\mathfrak{I}}, \quad(V, A) \mapsto V \mathrm{e}^{A},
$$

is a diffeomorphism.

Proof. For the Lie group structures of $\mathrm{GL}_{\mathfrak{I}}$ and $\mathrm{U}_{\mathfrak{I}}$ see e.g., Proposition 10.11 in [7]. We just recall that the topology of $\mathrm{GL}_{\mathfrak{I}}$ is defined by the metric $\left(V_{1}, V_{2}\right) \mapsto\left\|V_{1}-V_{2}\right\|_{\mathfrak{I}}$, where $\|\cdot\|_{\mathfrak{I}}$ is the norm of $\mathfrak{I}$.

The fact that the polar decomposition induces a diffeomorphism of $\mathrm{U}_{\mathfrak{I}} \times \mathfrak{p}_{\mathfrak{I}}$ onto $\mathrm{GL}_{\mathfrak{I}}$ follows just as in the special case $\mathfrak{I}=\mathfrak{S}_{p}$ treated in Proposition A.4 in [24].

Lemma 5.2 Let $\Phi$ be a symmetric norming function and $\mathfrak{I}=\mathfrak{S}_{\Phi}$. Also let $f:[0,1] \rightarrow \mathbb{R}$ be a continuous nondecreasing function such that $0 \leq f(t) \leq t$ whenever $t \in[0,1]$. Then for every sequence $\left\{A_{n}\right\}_{n \geq 1}$ in $\mathfrak{I}$ with $0 \leq A_{n} \leq 1$ for all $n \geq 1$ and $\lim _{n \rightarrow \infty}\left\|A_{n}\right\|_{\Phi}=0$ we have $f\left(A_{n}\right) \in \mathfrak{I}$ for all $n \geq 1$ and $\lim _{n \rightarrow \infty}\left\|f\left(A_{n}\right)\right\|_{\Phi}=0$.

Proof. We first recall from Remark 4.2(ii) that

$$
(\forall T \in \mathfrak{I}) \quad\|T\|_{\Phi}=\Phi\left(\left\{s_{j}(T)\right\}_{j \geq 1}\right) .
$$

Then for every positive integer $n$ we have

$$
\begin{aligned}
\left\|f\left(A_{n}\right)\right\|_{\Phi} & =\Phi\left(\left\{s_{j}\left(f\left(A_{n}\right)\right)\right\}_{j \geq 1}\right) & & \\
& =\Phi\left(\left\{f\left(s_{j}\left(A_{n}\right)\right)\right\}_{j \geq 1}\right) & & (\text { since } f \text { is nondecreasing }) \\
& \leq \Phi\left(\left\{s_{j}\left(A_{n}\right)\right\}_{j \geq 1}\right) & & (\text { since } 0 \leq f(t) \leq t \text { for } t \in[0,1]) \\
& =\left\|A_{n}\right\|_{\Phi} . & &
\end{aligned}
$$

Thus $\left\|f\left(A_{n}\right)\right\|_{\Phi}<\infty$ for all $n \geq 1$ and $\lim _{n \rightarrow \infty}\left\|f\left(A_{n}\right)\right\|_{\Phi}=0$. 
Theorem 5.3 Let $\Phi$ be a symmetric norming function, $\mathfrak{I}=\mathfrak{S}_{\Phi}, T=T^{*} \in \mathfrak{F}, \mathrm{U}_{\mathfrak{I}}(T):=\left\{V^{*} T V \mid V \in\right.$ $\left.\mathrm{U}_{\mathfrak{I}}\right\}$, and

$$
\pi: \mathrm{U}_{\mathfrak{I}} \rightarrow \mathrm{U}_{\mathfrak{I}}(T), \quad V \mapsto V^{*} T V .
$$

Then there exist an open neighborhood $\mathcal{D}$ of $T \in \mathcal{B}(\mathcal{H})$ and a map

$$
\varphi: \mathcal{D} \cap \mathrm{U}_{\mathfrak{I}}(T) \rightarrow \mathrm{U}_{\mathfrak{I}}
$$

such that

(i) $\varphi$ is continuous when $\mathcal{D} \cap \mathrm{U}_{\mathfrak{I}}(T)$ is equipped with the topology inherited from $\mathcal{B}(\mathcal{H})$ and $\mathrm{U}_{\mathfrak{I}}$ is equipped with its Lie group topology defined by the metric $\left(V_{1}, V_{2}\right) \mapsto\left\|V_{1}-V_{2}\right\|_{\Phi}$, and

(ii) $\pi \circ \varphi=\operatorname{id}_{\mathcal{D} \cap U_{\mathfrak{I}}(T)}$.

For the proof of this theorem we need some notations, remarks, and lemmas.

Notation 5.4 We now introduce some notation that will be used until the end of the proof of Theorem 5.3

(i) We denote $\sigma(T)=\left\{\lambda_{1}, \ldots, \lambda_{p}\right\}$, where $\lambda_{p}=0$.

(ii) For $i=1, \ldots, p$, we denote $\mathcal{K}_{i}=\operatorname{Ker}\left(T-\lambda_{i} \mathbf{1}\right), E_{i}$ the orthogonal projection of $\mathcal{H}$ onto $\mathcal{K}_{i}$, and $e_{i}$ is a polynomial in one variable with real coefficients such that $E_{i}=e_{i}(T)$.

(iii) We pick an open neighborhood $\mathcal{D}$ of $T \in \mathcal{B}(\mathcal{H})$ such that

$$
\max _{1 \leq i \leq p} \sup _{R \in \mathcal{D}}\left\|e_{i}(R)-e_{i}(T)\right\|<1 .
$$

Remark 5.5 Let $V \in \mathrm{U}_{\mathfrak{I}}$ with $R:=V^{*} T V \in \mathcal{D}$. For $i=1, \ldots, p$ we have

$$
\left\|V^{*} E_{i} V-E_{i}\right\|=\left\|V^{*} e_{i}(T) V-e_{i}(T)\right\|=\left\|e_{i}\left(V^{*} T V\right)-e_{i}(T)\right\|=\left\|e_{i}(R)-e_{i}(T)\right\|<1,
$$

whence

$$
\left\|\left(E_{i} V E_{i}\right)^{*}\left(E_{i} V E_{i}\right)-E_{i}\right\|<1 .
$$

On the other hand, the inequality $\left\|V^{*} E_{i} V-E_{i}\right\|<1$ also implies that

$$
\left\|E_{i}-V E_{i} V^{*}\right\| \leq\|V\|\left\|V^{*} E_{i} V-E_{i}\right\|\left\|V^{*}\right\|<1,
$$

whence

$$
\left\|\left(E_{i} V E_{i}\right)\left(E_{i} V E_{i}\right)^{*}-E_{i}\right\|<1 .
$$

Now (5.1) and (5.2) show that $\left.E_{i} V E_{i}\right|_{\mathcal{K}_{i}} \in \mathrm{GL}\left(\mathcal{K}_{i}\right)$ and thus we have a polar decomposition

$$
E_{i} V E_{i}=X_{i} Q_{i}
$$

with $Q_{i}=\left|E_{i} V E_{i}\right|=\left(\left(E_{i} V E_{i}\right)^{*}\left(E_{i} V E_{i}\right)\right)^{1 / 2}, \operatorname{Ker} X_{i}=\operatorname{Ker} Q_{i}=\mathcal{K}_{i}^{\perp}$, and $\left.X_{i}\right|_{\mathcal{K}_{i}} \in \mathrm{U}\left(\mathcal{K}_{i}\right)$. We will denote

$$
\psi(V)=X_{1}^{*}+\cdots+X_{p}^{*} \in \mathrm{U}(\mathcal{H})
$$

whenever $V \in \mathrm{U}_{\mathfrak{I}}$ is as above (that is, $V^{*} T V \in \mathcal{D}$ ). 
Notation 5.6 With the notation $\psi(\cdot)$ introduced in Remark [5.5] we define

$$
\varphi: \mathcal{D} \cap \mathrm{U}_{\mathfrak{I}}(T) \rightarrow \mathrm{U}(\mathcal{H}) \quad \text { by } \quad \varphi\left(V^{*} T V\right)=\psi(V) V,
$$

where $V \in \mathrm{U}_{\mathfrak{I}}$ and $V^{*} T V \in \mathcal{D}$.

Lemma 5.7 We have a well-defined map

$$
\varphi: \mathcal{D} \cap \mathrm{U}_{\mathfrak{I}}(T) \rightarrow \mathrm{U}_{\mathfrak{I}}
$$

satisfying $\pi \circ \varphi=\operatorname{id}_{\mathcal{D} \cap U_{\mathfrak{J}}(T)}$.

Proof. $\quad 1^{\circ}$ Let $R=V^{*} T V=W^{*} T W \in \mathcal{D}$ with $V, W \in \mathrm{U}_{\mathfrak{I}}$. Then $W V^{*} \in \mathrm{U}_{\mathfrak{I}} \cap\{T\}^{\prime}=\mathrm{U}_{\mathfrak{I}} \cap$ $\left\{E_{1}, \ldots, E_{p}\right\}^{\prime}$, so that (with the notation of Remark [5.5) we have

$$
E_{i} W E_{i}=W V^{*} E_{i} V E_{i}=\left(W V^{*} X_{i}\right) Q_{i},
$$

where $\left.W V^{*} X_{i}\right|_{\mathcal{K}_{i}} \in \mathrm{U}\left(\mathcal{K}_{i}\right)$ and $\operatorname{Ker}\left(W V^{*} X_{i}\right)=\operatorname{Ker} X_{i}=\operatorname{Ker} Q_{i}$. Thus the above equalities actually give the polar decomposition of $E_{i} W E_{i}$, whence

$$
\psi(W)=\sum_{i=1}^{p} X_{i}^{*} V W^{*}=\psi(V) V W^{*} .
$$

Consequently $\psi(W) W=\psi(V) V$, and thus the definition of $\varphi(R)$ is independent on the choice of $V \in \mathrm{U}_{\mathfrak{I}}$ with $R=V^{*} T V$.

$2^{\circ}$ We now check that $\varphi\left(V^{*} T V\right) \in \mathrm{U}_{\mathfrak{I}}$ if $V \in \mathrm{U}_{\mathfrak{I}}$ and $V^{*} T V \in \mathcal{D}$. First note that for all $i, j \in$ $\{1, \ldots, p\}$ we have $X_{i}, X_{j} \in\left\{E_{1}, \ldots, E_{p}\right\}^{\prime}$, hence $X_{i}^{*} X_{j}=X_{i}^{*} E_{i} X_{j}=\delta_{i j} E_{i}$ and similarly $X_{i} X_{j}^{*}=\delta_{i j} E_{i}$, where $\delta_{i j}$ is the Kronecker symbol. This implies that $\psi(V) \psi(V)^{*}=\psi(V)^{*} \psi(V)=\mathbf{1}$. Thus, in order to show that $\varphi(V)=\psi(V) V \in \mathrm{U}_{\mathfrak{I}}$, it remains to check that $\psi(V) \in \mathrm{U}_{\mathfrak{I}}$.

To this end, note that

$$
\delta(V):=\sum_{i=1}^{p} E_{i} V E_{i} \in \mathbf{1}+\mathfrak{I}
$$

since $\sum_{i=1}^{p} E_{i}=\mathbf{1}$ and $V \in \mathbf{1}+\mathfrak{I}$. On the other hand, as noted in Remark[5.5 we have $\left.E_{i} V E_{i}\right|_{\mathcal{K}_{i}} \in \mathrm{GL}\left(\mathcal{K}_{i}\right)$ for $i=1, \ldots, p$, hence $\delta(V) \in \mathrm{GL}(\mathcal{H})$ which proves that

$$
\delta(V) \in \mathrm{GL}_{\mathfrak{I}} .
$$

Since it is easy to see that the equality $\delta(V)=\psi(V)^{*}\left(Q_{1}+\cdots+Q_{p}\right)$ is just the polar decomposition of $\delta(V)$, it then follows by Lemma 5.1 that $\psi(V)^{*} \in \mathrm{U}_{\mathfrak{I}}$. Thus $\psi(V) \in \mathrm{U}_{\mathfrak{I}}$, as desired.

$3^{\circ}$ To finish the proof we have to show that, if $V \in \mathrm{U}_{\mathfrak{I}}$ and $V^{*} T V \in \mathcal{D}$, then $\pi\left(\varphi\left(V^{*} T V\right)\right)=V^{*} T V$. However, since $\psi(V) \in\{T\}^{\prime}$ and $\varphi\left(V^{*} T V\right)=\psi(V) V$, we have $\varphi\left(V^{*} T V\right)^{*} T \varphi\left(V^{*} T V\right)=V^{*} T V$, as required. 
Lemma 5.8 The map $\varphi: \mathcal{D} \cap \mathrm{U}_{\mathfrak{I}}(T) \rightarrow \mathrm{U}_{\mathfrak{I}}$ is continuous when $\mathcal{D} \cap \mathrm{U}_{\mathfrak{I}}(T)$ is equipped with the topology inherited from $\mathcal{B}(\mathcal{H})$ and $\mathrm{U}_{\mathfrak{I}}$ is equipped with its Lie group topology.

Proof. $1^{\circ}$ Let $\left\{V_{n}\right\}_{n \geq 1}$ be a sequence in $\mathrm{U}_{\mathfrak{I}}$ such that $\lim _{n \rightarrow \infty}\left\|V_{n}^{*} T V_{n}-T\right\|=0$. We will prove that $\lim _{n \rightarrow \infty}\left\|\varphi\left(V_{n}^{*} T V_{n}\right)-\mathbf{1}\right\|_{\Phi}=0$.

Clearly we may assume that $V_{n}^{*} T V_{n} \in \mathcal{D}$ for all $n \geq 1$. Denote $W_{n}=\varphi\left(V_{n}^{*} T V_{n}\right)$, so that $W_{n}^{*} T_{n} W_{n}=$ $V_{n}^{*} T V_{n}$ for all $n \geq 1$. Thus we also have $\lim _{n \rightarrow \infty}\left\|W_{n}^{*} T W_{n}-T\right\|=0$. Lemma 4.3 implies $\lim _{n \rightarrow \infty} \| W_{n}^{*} T W_{n}-$ $T \|_{\Phi}=0$.

For $i, j \in\{1, \ldots, p\}$ and $i \neq j$ we have $E_{i} T E_{i}=\lambda_{i} E_{i}$ and $E_{j} T E_{j}=\lambda_{j} E_{j}$, hence $E_{i}\left[T, W_{n}\right] E_{j}=$ $\left(\lambda_{i}-\lambda_{j}\right) E_{i} W_{n} E_{j}$. Then

$$
\left\|E_{i} W_{n} E_{j}\right\|_{\Phi} \leq \frac{\left\|T W_{n}-W_{n} T\right\|_{\Phi}}{\left|\lambda_{i}-\lambda_{j}\right|}=\frac{\left\|W_{n}^{*} T W_{n}-T\right\|_{\Phi}}{\left|\lambda_{i}-\lambda_{j}\right|},
$$

and thus $\lim _{n \rightarrow \infty}\left\|E_{i} W_{n} E_{j}\right\|_{\Phi}=0$.

Now let $i \in\{1, \ldots, p-1\}$, that is, $\lambda_{i} \neq 0$. Then $\lim _{n \rightarrow \infty}\left\|W_{n}^{*} T W_{n}-T\right\|=0$ implies $\lim _{n \rightarrow \infty} \| e_{i}\left(W_{n}^{*} T W_{n}\right)-$ $e_{i}(T) \|=0$, hence $\lim _{n \rightarrow \infty}\left\|W_{n}^{*} E_{i} W_{n}-E_{i}\right\|=0$. As in Remark [5.5] we get $\lim _{n \rightarrow \infty} \|\left(\begin{array}{c}n \rightarrow \infty \\ \stackrel{n}{n} W_{n} E_{i}\end{array}\right)^{*}\left(E_{i} W_{n} E_{i}\right)-$ $E_{i} \|=0$, that is, $\lim _{n \rightarrow \infty}\left\|E_{i}-\left(E_{i} W_{n} E_{i}\right)^{2}\right\|=0$. (Note that $E_{i} W_{n} E_{i}=E_{i} \varphi\left(V_{n}^{*} T V_{n}\right) E_{i} \geq 0$ according to Notation [5.6 and Remark [5.5) Since $\sup \left\{\operatorname{rank}\left(E_{i} W_{n} E_{i}\right) \mid n \geq 1\right\} \leq \operatorname{rank} E_{i}<\infty$ (here we use $\lambda_{i} \neq 0$ ), we get by Lemma 4.3 that $\lim _{n \rightarrow \infty}\left\|E_{i}-\left(E_{i} W_{n} E_{i}\right)^{2}\right\|_{\Phi}=0$. Now Lemma 5.2 applied for the function $f(t)=1-(1-t)^{1 / 2}$ shows that $\lim _{n \rightarrow \infty}\left\|E_{i}-E_{i} W_{n} E_{i}\right\|_{\Phi}=0$.

Next denote $A_{n}=\left(\mathbf{1}-E_{p}\right) W_{n}\left(\mathbf{1}-E_{p}\right), B_{n}=\left(\mathbf{1}-E_{p}\right) W_{n} E_{p}, C_{n}=E_{p} W_{n}\left(\mathbf{1}-E_{p}\right)$ and $D_{n}=$ $E_{p} W_{n} E_{p}$, so that

$$
W_{n}=\left(\begin{array}{cc}
A_{n} & B_{n} \\
C_{n} & D_{n}
\end{array}\right)
$$

in the sense that $W_{n}=A_{n}+B_{n}+C_{n}+D_{n}$. What we have already proved is that $\lim _{n \rightarrow \infty}\left(\left\|A_{n}-\left(\mathbf{1}-E_{p}\right)\right\|_{\Phi}+\right.$ $\left.\left\|B_{n}\right\|_{\Phi}+\left\|C_{n}\right\|_{\Phi}\right)=0$. Since $W_{n}^{*} W_{n}=\mathbf{1}$, we get $B_{n}^{*} B_{n}+D_{n}^{*} D_{n}=E_{p}$, so that $\lim _{n \rightarrow \infty}^{n \rightarrow \infty}\left\|D_{n}^{*} D_{n}-E_{p}\right\|_{\Phi}=0$. In other words, $\lim _{n \rightarrow \infty}\left\|E_{p}-\left(E_{p} W_{n} E_{p}\right)^{2}\right\|_{\Phi}=0$, whence $\lim _{n \rightarrow \infty}\left\|E_{p}-E_{p} W_{n} E_{p}\right\|_{\Phi}=0$ as above, by making use of Lemma 5.2 Consequently $\lim _{n \rightarrow \infty}\left\|W_{n}-\mathbf{1}\right\|_{\Phi}=0$, as desired.

$2^{\circ}$ We now prove that $\varphi: \mathcal{D} \cap \mathrm{U}_{\mathfrak{I}}(T) \rightarrow \mathrm{U}_{\mathfrak{I}}$ is continuous at all points of $\mathcal{D} \cap \mathrm{U}_{\mathfrak{I}}$. Let $\left\{V_{n}\right\}_{n \geq 1}$ be a sequence in $\mathrm{U}_{\mathfrak{I}}$ and $V \in \mathrm{U}_{\mathfrak{I}}$ such that $\lim _{n \rightarrow \infty}\left\|V_{n}^{*} T V_{n}-V^{*} T V\right\|=0$. We have to show that $\lim _{n \rightarrow \infty}\left\|\varphi\left(V_{n}^{*} T V_{n}\right)-\varphi\left(V^{*} T V\right)\right\|_{\Phi}=0$.

To this end, first note that $\lim _{n \rightarrow \infty}\left\|V V_{n}^{*} T V_{n} V^{*}-T\right\|=0$, hence $\lim _{n \rightarrow \infty}\left\|\varphi\left(V V_{n}^{*} T V_{n} V^{*}\right)-\mathbf{1}\right\|_{\Phi}=0$ by step $1^{\circ}$ of the proof. On the other hand, the operator $W_{n}:=\varphi\left(V V_{n}^{*} T V_{n} V^{*}\right)$ has the property $W_{n}^{*} T W_{n}=$ $V V_{n}^{*} T V_{n} V^{*}$, hence $V_{n}^{*} T V_{n}=V^{*} W_{n}^{*} T W_{n} V$, and thus $\varphi\left(V_{n}^{*} T V_{n}\right)=\varphi\left(V^{*} W_{n}^{*} T W_{n} V\right)=\psi\left(W_{n} V\right) W_{n} V$. We have $\lim _{n \rightarrow \infty}\left\|W_{n} V-V\right\|_{\Phi}=0$, hence it will suffice to show that $\lim _{n \rightarrow \infty}\left\|\psi\left(W_{n} V\right)-\psi(V)\right\|_{\Phi}=0$.

Thus we have to show that the map

$$
\psi: \pi^{-1}(\mathcal{D})\left(\subseteq \mathrm{U}_{\mathfrak{I}}\right) \rightarrow \mathrm{U}_{\mathfrak{I}}, \quad W \mapsto \psi(W),
$$


is continuous with respect to the topology of $U_{\mathfrak{I}}$. To see this, recall from step $2^{\circ}$ of the proof of Lemma 5.7 that, if $W \in \mathrm{U}_{\mathfrak{I}}$ and $W^{*} T W \in \mathcal{D}$, then $\delta(W)=\psi(W)^{*}|\delta(W)|$ is the polar decomposition of $\delta(W) \in \mathrm{GL}_{\mathfrak{I}}$. Now Lemma 5.1 along with the obvious continuity of the map $\delta: \pi^{-1}(\mathcal{D}) \rightarrow \mathrm{GL}_{\mathfrak{I}}$ imply that the map $\psi: \pi^{-1}(\mathcal{D}) \rightarrow \mathrm{U}_{\mathfrak{I}}$ is continuous.

Proof of Theorem [5.3. Just use Lemmas 5.7 and 5.8 (see also Notation [5.4).

Lemma 5.9 Let $U, Q, Q_{1}$ be topological spaces, $p: U \rightarrow Q$ and $\iota: Q \rightarrow Q_{1}$ continuous mappings, and $p_{1}:=\iota \circ p$. Assume that the following conditions are satisfied:

(i) The map $\iota$ is injective.

(ii) For every $x_{1} \in Q_{1}$ there exist a neighborhood $W_{1}$ of $x_{1}$ and a continuous map $\sigma_{1}: W_{1} \rightarrow U$ such that $p_{1} \circ \sigma_{1}=\operatorname{id}_{W_{1}}$.

Then $\iota$ is a homeomorphism of $Q$ onto $Q_{1}$.

Proof. We have by (ii) that the map $p_{1}$ is onto. Since $\iota \circ p=p_{1}$, it then follows that $\iota$ is onto as well. Thus it only remains to show that $\iota^{-1}: Q_{1} \rightarrow Q$ is continuous.

To this end, let $x_{1} \in Q_{1}$ arbitrary. According to hypothesis (ii), there is a continuous map $\sigma_{1}: W_{1} \rightarrow$ $U$ on some neighborhood $W_{1}$ of $x_{1}$ such that $p_{1} \circ \sigma_{1}=\mathrm{id}_{W_{1}}$, that is, $\iota \circ p \circ \sigma_{1}=\mathrm{id}_{W_{1}}$. Then $\left.\iota^{-1}\right|_{W_{1}}=p \circ \sigma_{1}$, hence $\iota^{-1}$ is continuous on the neighborhood $W_{1}$ of $x_{1}$. Since $x_{1} \in Q_{1}$ was arbitrary, it follows that $\iota^{-1}$ is continuous on the whole set $Q_{1}$.

Concerning part (i) in the statement of the next theorem, we note that it involves two (completely unrelated to each other) symmetric norming functions. On the topological level, this corresponds to the fact that any two symmetric norming functions define the same topology (in fact, the norm topology) on any unitary orbit of a finite-rank operator, as a consequence of Lemma 4.3. We should point out that there exist a large variety of symmetric norming functions, defining various types of operator ideals like Schatten, Lorentz, Orlicz and so on (see [13] for a survey of this subject). By way of illustrating this remark, we recall that we have already mentioned in Remark 4.2(iii) the functions $\Phi_{p}(\cdot)=\|\cdot\|_{\ell^{p}}$ that define the Schatten ideals. For other concrete symmetric norming functions, see Example 5.11 below.

Theorem 5.10 Let $\Phi$ and $\Psi$ be symmetric norming functions, $\mathfrak{I}=\mathfrak{S}_{\Phi}, T=T^{*} \in \mathfrak{F}$ and $\mathrm{U}_{\mathfrak{I}}(T):=$ $\left\{V^{*} T V \mid V \in \mathrm{U}_{\mathfrak{I}}\right\}$. Then the following assertions hold:

(i) The orbit map

$$
\pi: \mathrm{U}_{\mathfrak{I}} \rightarrow \mathfrak{F}, \quad V \mapsto V^{*} T V
$$

induces a diffeomorphism of the homogeneous space $\mathrm{U}_{\mathfrak{I}} / \mathrm{U}_{\mathfrak{I}, T}$ onto the submanifold $\mathrm{U}_{\mathfrak{I}}(T)$ of $\mathfrak{S}_{\Psi}$.

(ii) If moreover $\Psi^{*}=\Phi$ and the Banach Lie group $\mathrm{U}_{\mathfrak{I}}$ is connected, then the orbit $\mathrm{U}_{\mathfrak{I}}(T)$ is a $\mathrm{U}_{\mathfrak{I}}$ homogeneous weakly Kähler manifold. 
Proof. (i) We first use Lemma 5.9 with $U=\mathrm{U}_{\mathfrak{I}}, Q=\mathrm{U}_{\mathfrak{I}} / \mathrm{U}_{\mathfrak{I}, T}, Q_{1}=\mathrm{U}_{\mathfrak{I}}(T), p: \mathrm{U}_{\mathfrak{I}} \rightarrow \mathrm{U}_{\mathfrak{I}} / \mathrm{U}_{\mathfrak{I}, T}$ the quotient map and $\iota: \mathrm{U}_{\mathfrak{I}} / \mathrm{U}_{\mathfrak{I}, T} \rightarrow \mathrm{U}_{\mathfrak{I}}(T)$ induced by the orbit map $\pi$, to deduce that the differentiable map $\iota$ is a homeomorphism, hence a diffeomorphism. Note that condition (ii) in Lemma [5.9 is satisfied as a consequence of Theorem [5.3. In order to prove that $\mathrm{U}_{\mathfrak{I}}(T)$ is an embedded submanifold of $\mathfrak{S}_{\Psi}$, we now show that the weak immersion $\iota: \mathrm{U}_{\mathfrak{I}} / \mathrm{U}_{\mathfrak{I}, T} \rightarrow \mathfrak{S}_{\Psi}$ is actually an immersion. To this end note that the range of its differential at the point $p(\mathbf{1}) \in \mathrm{U}_{\mathfrak{I}} / \mathrm{U}_{\mathfrak{I}, T}$ is

$$
\left\{[T, Y] \mid Y \in \mathfrak{u}_{\mathfrak{I}}\right\}=\left\{[T, Y] \mid Y=-Y^{*} \in \mathfrak{F}\right\}=\left\{[T, Y] \mid Y=-Y^{*} \in \mathfrak{S}_{1}\right\},
$$

and this is a closed complemented subspace of $\mathfrak{S}_{\Psi}$, as an easy consequence of Theorem 3.2 and Lemma 4.3

(ii) Just use Proposition 4.8 along with Remark 4.2(iii) (see also the equality (4.2) in step $1^{\circ}$ in the proof of Proposition 4.8.

Example 5.11 Let $\Pi=\left\{\pi_{j}\right\}_{j \geq 1}$ be a sequence of real numbers satisfying the conditions

(i) $1=\pi_{1} \geq \pi_{2} \geq \cdots>0$, and

(ii) $\sum_{j=1}^{\infty} \pi_{j}=\infty$.

Let $\mathcal{K}(\mathcal{H})$ denote the ideal of compact operators on $\mathcal{H}$ and define

$$
\begin{aligned}
& \mathfrak{S}_{\pi}=\left\{A \in \mathcal{K}(\mathcal{H}) \mid\|A\|_{\pi}:=\sum_{j=1}^{\infty} \pi_{j} \mathrm{~s}_{j}(A)<\infty\right\}, \\
& \mathfrak{S}_{\Pi}=\left\{A \in \mathcal{B}(\mathcal{H}) \mid\|A\|_{\Pi}:=\sup _{n \geq 1} \frac{\mathrm{s}_{1}(A)+\cdots+\mathrm{s}_{n}(A)}{\pi_{1}+\cdots+\pi_{n}}<\infty\right\},
\end{aligned}
$$

where $\left(\mathrm{s}_{j}(A)\right)_{j \geq 1}$ denotes, as usual, the sequence of singular numbers of an operator $A \in \mathcal{B}(\mathcal{H})$ (see e.g., Remark 4.2 (ii)). In other words, $\mathfrak{S}_{\pi}=\mathfrak{S}_{\Phi_{\pi}}=\mathfrak{S}_{\Phi_{\pi}}^{(0)}$ and $\mathfrak{S}_{\Pi}=\mathfrak{S}_{\Phi_{\Pi}}$, where the symmetric norming functions $\Phi_{\pi}, \Phi_{\Pi}: \widehat{c} \rightarrow \mathbb{R}$ are defined by

$$
\Phi_{\pi}(\xi)=\sum_{j=1}^{\infty} \pi_{j} \xi_{j} \quad \text { and } \quad \Phi_{\Pi}(\xi)=\sup _{n \geq 1} \frac{\xi_{1}+\cdots+\xi_{n}}{\pi_{1}+\cdots+\pi_{n}}
$$

whenever $\xi=\left\{\xi_{j}\right\}_{j \geq 1} \in \widehat{c}$ and $\xi_{1} \geq \xi_{2} \geq \cdots \geq 0$. We note that $\left(\Phi_{\pi}\right)^{*}=\Phi_{\Pi}$ by the comments preceding Theorem 15.2 in [15]. It then follows by Theorem 15.2 in [15] that $\left(\mathfrak{S}_{\pi}, \mathfrak{S}_{\Pi}\right)$ is a pair of Banach ideals satisfying the hypotheses of Proposition 4.8

If moreover the sequence $\Pi=\left\{\pi_{j}\right\}_{j \geq 1}$ is regular, in the sense that it satisfies the condition

(iii) $\sup _{n \geq 1}\left(\sum_{j=1}^{n} \pi_{j}\right) /\left(n \pi_{n}\right)<\infty$, 
then we have the equality $\mathfrak{S}_{\Pi}=\left\{A \in \mathcal{B}(\mathcal{H}) \mid \mathrm{s}_{n}(A)=O\left(\pi_{n}\right)\right.$ as $\left.n \rightarrow \infty\right\}$ according to Theorem 14.2 in [15.

We note that, just as in the special case of the similar pair $\left(\mathfrak{S}_{1}, \mathcal{B}(\mathcal{H})\right)$, the dual space $\mathfrak{S}_{\Pi}$ is in general a non-separable Banach space (see Theorem 14.1 in [15] and Remark 4.2[iii)).

For the sake of completeness, we note that in the case when the sequence $\Pi$ is constant, that is, $\pi_{1}=\pi_{2}=\cdots=1$, we get $\mathfrak{S}_{\pi}=\mathfrak{S}_{1}$ the trace class, and $\mathfrak{S}_{\Pi}=\mathcal{B}(\mathcal{H})$.

This is precisely the situation when the above Theorem[5.10)(i) reduces to Theorem 2.5 in 9 ] (a part of its proof appears already in [8]). That is, to get the latter result, we have to apply Theorem [5.10(i) for $\mathfrak{I}=\mathcal{B}(\mathcal{H})$, i.e., $\Phi\left(\left\{\xi_{j}\right\}_{j \geq 1}\right)=\max _{j \geq 1}\left|\xi_{j}\right|$ and $\Psi\left(\left\{\xi_{j}\right\}_{j \geq 1}\right)=\sum_{j=1}^{\infty}\left|\xi_{j}\right|$.

Acknowledgments. We thank P. Bona for a number of useful remarks and also for sending us the preprint of his paper 9 that was one of our motivations for this work. We thank the referee, K.-H. Neeb, A. Odzijewicz, M. Rieffel, and G. Weiss for their comments that influenced some of our presentation. The first author was partially supported by grant CERES 3-28/2003, and by the Swiss NSF through the SCOPES Program during a one month visit at the EPFL; the excellent working conditions provided by EPFL are gratefully acknowledged. The second author was partially supported by the European Commission and the Swiss Federal Government through funding for the Research Training Network Mechanics and Symmetry in Europe (MASIE) as well as the Swiss National Science Foundation.

\section{References}

[1] E. Andruchow, D. Stojanoff, Differentiable structure of similarity orbits J. Operator Theory, 21(2) (1989), 349-366.

[2] E. Andruchow, D. Stojanoff, Geometry of unitary orbits, J. Operator Theory, 26(1) (1991), 25-41.

[3] E. Andruchow, A. Varela, Weight centralizer expectations with finite index, Math. Scand., 84(2) (1999), 243-260

[4] E. Andruchow, A. Varela, Homotopy of state orbits, J. Operator Theory, 48(2) (2002), 419-430.

[5] C. Apostol, Inner derivations with closed range, Rev. Roumaine Math. Pures Appl., 21(3) (1976), $249-265$

[6] C. Apostol, L.A. Fialkow, D.A. Herrero, D. Voiculescu, Approximation of Hilbert Space Operators, vol II, Research Notes in Mathematics, 102. Pitman (Advanced Publishing Program), Boston, MA, 1984 .

[7] D. Beltiţă, Equivariant monotone operators and infinite-dimensional complex homogeneous spaces, Preprint IMAR, 2002.

[8] P. Bona, Extended quantum mechanics. Acta Physica Slovaca, 50(1) (2000), 1 - 198.

[9] P. Bona, Some considerations on topologies of infinite dimensional unitary coadjoint orbits, $J$. Geom. Phys. 51(2) (2004), 256-268. 
[10] F.F. Bonsall, J. Duncan, Numerical Ranges of Operators on Normed Spaces and of Elements of Normed Algebras, London Mathematical Society Lecture Note Series, 2. Cambridge University Press, London-New York, 1971.

[11] N. Bourbaki, Groupes et algèbres de Lie, Chapitre 3, Paris: Hermann, 1972.

[12] D. Deckard, L.A. Fialkow, Characterization of Hilbert space operators with unitary cross sections, J. Operator Theory 2(2) (1979), 153-158.

[13] K. Dykema, T. Figiel, G. Weiss, M. Wodzicki, Commutator structure of operator ideals, Adv. in Math. 185(1) (2004), 1-79.

[14] L. Fialkow, R. Loebl, Elementary mappings into ideals of operators, Illinois J. Math., 28(4) (1984), $555-578$.

[15] I.C. Gohberg, M.G. Kreun, Introduction to the Theory of Linear Nonselfadjoint Operators, Translations of Mathematical Monographs, vol. 18, American Mathematical Society, Providence, R.I., 1969 .

[16] F. Greenleaf, Invariant Means on Topological Groups and Their Applications, van NostrandReinhold Co., New York-Toronto-London-Melbourne, 1969.

[17] V. Guillemin and S. Sternberg, Symplectic Techniques in Physics. Cambridge University Press, Cambridge, 1984.

[18] L.A. Harris, W. Kaup, Linear algebraic groups in infinite dimensions, Illinois J. Math. 21(3) (1977), 666-674.

[19] D.A. Herrero, Approximation of Hilbert Space Operators, vol. 1, Second edition, Pitman Research Notes in Mathematics Series, 224, Longman Scientific \& Technical, Harlow, copublished in the United States with John Wiley \& Sons, New York, 1989.

[20] V. Jones, V.S. Sunder, Introduction to Subfactors, London Mathematical Society Lecture Note Series, 234, Cambridge University Press, Cambridge, 1997.

[21] L. Kérchy, Generalized Toeplitz operators, Acta Sci. Math. (Szeged) 68(1-2) (2002), 373-400.

[22] J.E. Marsden, T.S. Ratiu, Introduction to Mechanics and Symmetry. A Basic Exposition of Classical Mechanical Systems, second edition, Texts in Applied Mathematics, 17, Springer-Verlag, New York, 1999 second printing 2003.

[23] K. Mattila, Normal operators and proper boundary points of the spectra of operators on a Banach space, Ann. Acad. Sci. Fenn. Ser. A I Math. Dissertationes 19 (1978), 48 pp.

[24] K.-H. Neeb, Infinite-dimensional groups and their representations, in: Lie Theory, Progr. Math. 228, Birkhäuser, Boston, MA, 2004, pp. 213-328. 
[25] K.-H. Neeb, Central extensions of infinite-dimensional Lie groups, Ann. Inst. Fourier. 52(5) (2002), $1365-1442$.

[26] K.-H. Neeb and C. Vizman, Flux homomorphisms and principal bundles over infinite-dimensional manifolds, Monatshefte für Math. 139 (2003), 309-333.

[27] K.-H., Neeb, Abelian extensions of infinite-dimensional Lie groups, preprint, arXiv: math.GR/0402303

[28] A. Odzijewicz, T.S. Ratiu, Banach Lie-Poisson spaces and reduction, Comm. Math. Phys. 243 (2003), 1-54.

[29] R.S. Palais, On the homotopy type of certain groups of operators, Topology 3 (1965), 271-279.

[30] M. Pecuch Herrero, Global cross sections of unitary and similarity orbits of Hilbert space operators, J. Operator Theory, 12(2) (1984), 265-283.

[31] G.K. Pedersen, $C^{*}$-algebras and Their Automorphism Groups, Academic Press, London - New York - San Francisco, 1979.

[32] Ş. Strătilă, L. Zsidó, Lectures on von Neumann Algebras, Editura Academiei - Abacus Press, Bucharest - Tunbridge Wells, 1979.

[33] M. Takesaki, Conditional expectations in von Neumann algebras, J. Functional Analysis 9 (1972), $306-321$.

[34] D.V. Voiculescu, K.J. Dykema, A. Nica, Free Random Variables, CRM Monograph Series, vol. 1, Amer. Math. Soc., 1992.

[35] A. Weinstein, The local structure of Poisson manifolds, J. Differential Geom. 18(3) (1983), 523-557. 